\title{
Article \\ Catalase (CAT) Gene Family in Rapeseed (Brassica napus L.): Genome-Wide Analysis, Identification, and Expression Pattern in Response to Multiple Hormones and Abiotic Stress Conditions
}

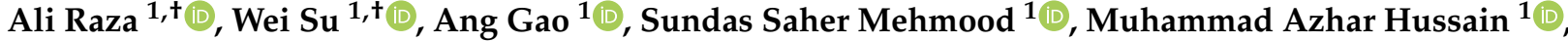 \\ Wenlong $\mathrm{Nie}^{2}$, Yan Lv ${ }^{1, * \mathbb{D}}$, Xiling Zou ${ }^{1, *(D)}$ and Xuekun Zhang ${ }^{3}$
}

1 Key Lab of Biology and Genetic Improvement of Oil Crops, Oil Crops Research Institute, Chinese Academy of Agricultural Sciences (CAAS), Wuhan 430062, China; alirazamughal143@gmail.com (A.R.); 82101181063@caas.cn (W.S.); gaoang1696555087@163.com (A.G.); snookas.saher90@gmail.com (S.S.M.); azharhussain301@gmail.com (M.A.H.)

2 Crops Research Laboratory, Xiaogan Academy of Agricultural Sciences, Xiaogan 432000, China; 15871339003@163.com

3 College of Agriculture, Engineering Research Center of Ecology and Agricultural Use of Wetland of Ministry of Education, Yangtze University, Jingzhou 434025, China; zhang.xk@139.com

* Correspondence: lvyan01@caas.cn (Y.L.); zouxiling01@caas.cn (X.Z.)

Citation: Raza, A.; Su, W.; Gao, A.; Mehmood, S.S.; Hussain, M.A.; Nie, W.; Lv, Y.; Zou, X.; Zhang, X. Catalase (CAT) Gene Family in Rapeseed (Brassica napus L.):

Genome-Wide Analysis,

Identification, and Expression Pattern in Response to Multiple Hormones and Abiotic Stress Conditions. Int. J. Mol. Sci. 2021, 22, 4281. https:// doi.org/10.3390/ijms22084281

Academic Editor: Hon-Ming Lam

Received: 2 April 2021

Accepted: 19 April 2021

Published: 20 April 2021

Publisher's Note: MDPI stays neutral with regard to jurisdictional claims in published maps and institutional affiliations.

Copyright: () 2021 by the authors. Licensee MDPI, Basel, Switzerland. This article is an open access article distributed under the terms and conditions of the Creative Commons Attribution (CC BY) license (https:// creativecommons.org/licenses/by/ $4.0 /)$.
+ These authors contributed equally to this work.

\begin{abstract}
Catalase (CAT) is an antioxidant enzyme expressed by the CAT gene family and exists in almost all aerobic organisms. Environmental stresses induce the generation of reactive oxygen species (ROS) that eventually hinder plant growth and development. The CAT enzyme translates the hydrogen peroxide $\left(\mathrm{H}_{2} \mathrm{O}_{2}\right)$ to water $\left(\mathrm{H}_{2} \mathrm{O}\right)$ and reduce the ROS levels to shelter the cells' death. So far, the CAT gene family has not been reported in rapeseed (Brassica napus L.). Therefore, a genomewide comprehensive analysis was conducted to classify the $C A T$ genes in the rapeseed genome. The current study identified $14 B n C A T$ genes in the rapeseed genome. Based on phylogenetic and synteny analysis, the BnCATs belong to four groups (Groups I-IV). A gene structure and conserved motif analysis showed that Group I, Group II, and Group IV possess almost the same intron/exon pattern, and an equal number of motifs, while Group III contains diverse structures and contain 15 motifs. By analyzing the cis-elements in the promoters, we identified five hormone-correlated responsive elements and four stress-related responsive elements. Further, six putative bna-miRNAs were also identified, targeting three genes (BnCAT4, BnCAT6, and BnCAT8). Gene ontology (GO) enrichment analysis showed that the $B n C A T$ genes were largely related to cellular organelles, ROS response, stimulus response, stress response, and antioxidant enzymes. Almost $10 \mathrm{BnCAT}$ genes showed higher expression levels in different tissues, i.e., root, leaf, stem, and silique. The expression analysis showed that $B n C A T 1-B n C A T 3$ and $B n C A T 11-B n C A T 13$ were significantly upregulated by cold, salinity, abscisic acid (ABA), and gibberellic acid (GA) treatment, but not by drought and methyl jasmonate (MeJA). Notably, most of the genes were upregulated by waterlogging stress, except $B n C A T 6, B n C A T 9$, and BnCAT10. Our results opened new windows for future investigations and provided insights into the $C A T$ family genes in rapeseed.
\end{abstract}

Keywords: abiotic stress; catalase; developmental tissues; gene structure; gene ontology; miRNA; plant hormones; ROS; stress responses

\section{Introduction}

Numerous environmental factors, including abiotic and biotic stresses, significantly limit plants' growth and development [1]. Under stress environments, the plant modifies its homeostatic apparatus by producing a surplus level of reactive oxygen species (ROS). 
ROS, which consist of a superoxide anion, hydrogen peroxide, hydroxyl radical, and singlet oxygen, are produced as a lethal derivative of regular oxygen metabolism and serve as a signaling component in plant molecular biology [2,3]. Over the past decade, many investigations have discovered that ROS homeostasis is important for upholding normal cellular roles [2,3]. Therefore, upholding a considerable amount of ROS is crucial for appropriate cellular ROS signaling, and this amount can be attained by maintaining the balance among ROS generation and scavenging [2,3].

The major ROS-metabolizing enzymes include superoxide dismutase, catalase, peroxidase, glutathione peroxidase, ascorbate peroxidase, glutathione reductase, glutathione S-transferase, etc., among which the catalase (CAT; EC 1.11.1.6) enzyme is crucial for ROS depollution throughout stress, and it does not need cellular reductants as they mainly catalyze a dismutase retort [4]. The tetrameric haem-comprising CAT enzyme quickly decays $\mathrm{H}_{2} \mathrm{O}_{2}$, producing water $\left(\mathrm{H}_{2} \mathrm{O}\right)$ and oxygen $\left(\mathrm{O}_{2}\right)$, and exists in almost all aerobic organisms [4]. The activity of CAT has been detected in peroxisomes, mitochondria, cytosol, and chloroplast [2,4]. Notably, CAT owns the extreme turnover proportion, and 26 million $\mathrm{H}_{2} \mathrm{O}_{2}$ molecules can be changed by one CAT molecule within one minute [5,6]. In animals, CAT enzymes are programmed by an exclusive CAT gene. In contrast, in plants, these enzymes are programmed by a gene family and have been systematically investigated in a number of plant species. For instance, one CAT family member (CAT1) has been reported in castor bean (Ricinus communis L.) [7], tomato (Lycopersicon esculentum) [8], and sweet potato (Ipomoea batatas L.) [9]; two CAT members (CAT1 and CAT2) in barley (Hordeum vulgare L.) [10]; three CAT members (CAT1-CAT3) in tobacco (Nicotiana plumbaginifolia) [11], maize (Zea mays L.) [12], pumpkin (Cucurbita Linn.) [13], A. thaliana [14], and rice (Oryza sativa L.) [15]; and four (CAT1-CAT4) in cucumber (Cucumis sativus L.) [16] and rice [17]. So far, the highest number (7) of CAT genes has been documented in cotton (Gossypium hirsutum L. and G. barbadense L.) [18].

Several investigations have recommended that CAT genes' expression is modulated temporally and spatially and reacts to numerous environmental pressures [9,19-23]. In A. thaliana, $C A T 1$ is a vital gene that responds to numerous abiotic stresses by scavenging $\mathrm{H}_{2} \mathrm{O}_{2}$. Moreover, CAT2 and CAT3 are involved in eliminating $\mathrm{H}_{2} \mathrm{O}_{2}$ subsidized to ROS homeostasis in light or dark conditions [4]. According to Du et al. [14], CAT2 could be triggered in response to drought and cold stresses, while CAT3 was primarily triggered by abscisic acid (ABA) and oxidative treatments at the senescence phase. In $A$. thaliana, it has been validated that CAT2 is the main isoform in the leaf, which is systematically connected with photorespiration [24]. Additionally, Contento and Bassham [25] reported that $A$. thaliana cat 2 mutants show areas of chlorosis and necrotic scratches. In Ipomoea batatas, the transcript level of CAT1 was persuaded via ethephon and stimulated by condensed glutathione (GSH), nicotinamide adenine dinucleotide phosphate (NADPH) oxidase inhibitor diphenylene iodonium (DPI), calcium ion $\left(\mathrm{Ca}^{2+}\right)$ chelator egtazic acid, and cycloheximide [9]. Moreover, transgenic N. tabacum plants carrying the CAT2 gene from maize showed improved tolerance to pathogen contagion and oxidative stress [26].

Likewise, the expression levels of the CAT genes vary in different tissues. For instance, in hot pepper (Capsicum annuum L.), the transcript levels of CAT1-CAT3 in diverse organs (i.e., leaf and stem) are linked to circadian rhythms and different stresses [27]. In tobacco, CAT1 and CAT2 are spotted in non-senescent leaves; the transcript pattern of CAT2 is expressively decreased in senescent leaves. In contrast, CAT3 is spotted in both types of plants [28]. In Scots pine (Pinus sylvestris L.), CAT is elaborated in embryogenesis and cell death procedures [29]. In cucumber, CAT1-CAT3 express in different organs (root, stem, leaves, flowers, and fruits), and could be significantly induced by salinity, cold, drought, and $\mathrm{ABA}$ treatments [16]. 
Moreover, previous investigations advised that miRNA-mediated modulation of ROSassociated genes is imperative for plant growth and development [30,31], as well as for stress resistance [32-35]. In Chinese cottonwood (Populus simonii), the interaction network between DNA methylation and miRNA (Ptc-miR396s and Ptc-miR156s)-target genes, and their products, affect the metabolic aspects of the antioxidant-encoding genes such as $S O D$ and CAT under temperature stress. Results show that DNA methylation possibly modulates the transcript levels of the miRNA genes, therefore distressing the transcript of their target genes [36]. Likewise, Wang et al. [18] prophesied the miRNA-facilitated modulation of cotton CAT genes and detected some presumed target positions (catalase domain) of cotton miRNAs (novel_mir_2537/0819, miRCS46a, miRCS46a/gb-miR7486, and ghr-miR6138, etc.) under Verticillium dahlia infection. These findings specified that miRNAs might play vital roles in response to environmental cues via modulating the CAT genes.

Until now, CAT genes have been documented in several plant species and investigated under different stress conditions. Particularly, the CAT gene family has not been reported in rapeseed. Rapeseed (Brassica napus L.) is considered the second major oilseed crop after soybean (Glycine max L.). It has a composite genome because of its evolutionary past. Rapeseed yield restrictions have been changed owing to the augmented experience of multiple abiotic stresses [37-39]. Therefore, a genome-wide comprehensive investigation has been carried out to identify the CAT gene family in rapeseed for the first time. Further, their phylogenetic relationships, synteny analysis, gene structures, conserved motifs, ciselements, miRNA regulator prediction, and functional annotation have been characterized. Furthermore, the expression pattern in different tissues and under various hormone and abiotic stresses has been widely evaluated to get insight into the rapeseed $C A T$ genes.

\section{Results}

\subsection{Identification of CAT Genes Family in Rapeseed}

In the present study, a total of $14 C A T$ genes were recognized in the complete genome of rapeseed via BLASTP using 3 AtCAT protein sequences as queries (Table 1). These genes were named $B n C A T 1-B n C A T 14$, among which seven genes (BnCAT1-BnCAT7) were positioned in the A subgenome, and five genes (BnCAT8-BnCAT12) were positioned in the $C$ subgenome (Table 1). BnCAT13 and BnCAT14 are distributed in two scaffold regions (Bnascaffold0025 and Bnascaffold0025) and have not yet been incorporated into the physical map of chromosomes (Table 1). Detailed information of the 14 BnCAT genes is presented in Table 1. Briefly, gene length ranged from $1990 \mathrm{bp}$ (BnCAT6) to $9770 \mathrm{bp}$ (BnCAT10) with four to five exons in each sequence. Notably, BnCAT5 comprises six exons, and three genes (BnCAT3, BnCAT9, and BnCAT10) consisted of 12 and 15 exons, respectively. The coding DNA sequences (CDS) ranged from $1434 \mathrm{bp}$ (BnCAT13) to 3123 bp (BnCAT10), while the protein length varied from 477 (BnCAT13) to 1040 (BnCAT10) amino acids. The prophesied molecular weights of the $14 \mathrm{BnCAT}$ proteins ranged from $55.19 \mathrm{kDa}(B n C A T 13)$ to $565.40 \mathrm{kDa}$ (BnCAT6), and the isoelectric points extended from 6.5 (BnCAT1) to 8.42 (BnCAT5). The subcellular localization outcomes predicted that nine BnCAT proteins were positioned on the peroxisome, while the remaining proteins were located on cytoskeleton (BnCAT1), cytoplasm (BnCAT4), mitochondrion (BnCAT9), and chloroplast (BnCAT5 and BnCAT10) (Table 1). Meanwhile, 4 and 7 CAT genes were also identified from the Brassica oleracea (BoCAT1-BoCAT4) and Brassica rapa (BraCAT1-BraCAT7) genomes, respectively (Table S2). 
Table 1. Information of the 14 BnCAT genes identified in rapeseed.

\begin{tabular}{|c|c|c|c|c|c|c|c|c|c|}
\hline Gene ID & Gene Name & Genomic Position (bp) & $\begin{array}{l}\text { Gene Length } \\
\text { (bp) }\end{array}$ & $\begin{array}{l}\text { CDS Length } \\
\text { (bp) }\end{array}$ & Exon & $\begin{array}{l}\text { Protein Length } \\
\text { (Amino Acids) }\end{array}$ & $\begin{array}{c}\text { Molecular } \\
\text { Weight (kDa) }\end{array}$ & $\begin{array}{c}\text { Isoelectric } \\
\text { Point (pI) }\end{array}$ & $\begin{array}{l}\text { Sub-Cellular } \\
\text { Localization }\end{array}$ \\
\hline BnaA01T0032600ZS & BnCAT1 & A01:1801796-1803991 + & 2195 & 1488 & 4 & 495 & 57.30 & 6.5 & Cytoskeleton \\
\hline BnaA03T0547900ZS & $B n C A T 2$ & A03:31026573-31028922 - & 2349 & 1479 & 5 & 492 & 56.84 & 6.63 & Peroxisome \\
\hline BnaA06T0144600ZS & $B n C A T 3$ & A06:8685726-8691515 + & 5789 & 2925 & 12 & 974 & 112.30 & 6.78 & Peroxisome \\
\hline BnaA07T0132000ZS & BnCAT4 & A07:17121532-17124087 - & 2555 & 1479 & 5 & 492 & 56.90 & 6.83 & Cytoplasm \\
\hline BnaA07T0132100ZS & BnCAT5 & A07:17124704-17130749 - & 6045 & 1671 & 6 & 556 & 64.83 & 8.42 & Chloroplast \\
\hline BnaA08T0247000ZS & $B n C A T 7$ & A08:25020319-25023539 - & 3220 & 1479 & 5 & 492 & 56.80 & 7.69 & Peroxisome \\
\hline BnaC03T0740800ZS & BnCAT8 & C03:71851456-71853466 + & 2010 & 1470 & 4 & 489 & 56.52 & 6.77 & Peroxisome \\
\hline BnaC05T0176000ZS & BnCAT9 & C05:11786858-11795441+ & 8583 & 2949 & 12 & 982 & 113.09 & 6.94 & Mitochondrion \\
\hline BnaC07T0194100ZS & BnCAT10 & C07:32771906-32781676 - & 9770 & 3123 & 15 & 1040 & 120.71 & 7.34 & Chloroplast \\
\hline BnaC07T0524400ZS & BnCAT11 & C07:59429784-59432167 - & 2383 & 1479 & 4 & 492 & 56.78 & 6.63 & Peroxisome \\
\hline BnaC08T0260800ZS & BnCAT12 & C08:34921906-34925039+ & 3133 & 1479 & 5 & 492 & 56.80 & 7.69 & Peroxisome \\
\hline Bnascaffold0026T0027200ZS & BnCAT14 & 0026:2854478-2857610 + & 3132 & 1479 & 5 & 492 & 56.80 & 7.69 & Peroxisome \\
\hline
\end{tabular}

In the genomic position, the positive (+) and negative (-) signs indicate the existence of a gene on the positive and negative strand of that specific marker, respectively. 


\subsection{Phylogenetic Relationships of CAT Genes}

To explore the evolutionary relationships between the BnCAT, BoCAT, BraCAT, and AtCAT genes, an unrooted phylogenetic tree was constructed, which was clustered into four groups (Groups I-IV) (Figure 1). Our results showed that Group I comprised of 7 CAT members (4 BnCATs, 2 BraCATs, and 1 BoCAT); Group II comprised of 4 CAT members (3 $B n C A T s$ and 1 BoCAT); Group III comprised of 7 CAT members (2 BnCATs, 2 BraCATs, 1 $B o C A T$, and 2 AtCATs); and Group IV comprised of $10 C A T$ members (5 BnCATs, 3 BraCATs, $1 B o C A T$, and $1 A t C A T)$. Notably, the $B n C A T$ genes were distributed in four groups, and Group IV was found to contain more BnCATs than the other three groups (Figure 1). Furthermore, it was found that the BnCATs have a closer phylogenetic relationship with the BoCATs and BraCATs in each group.

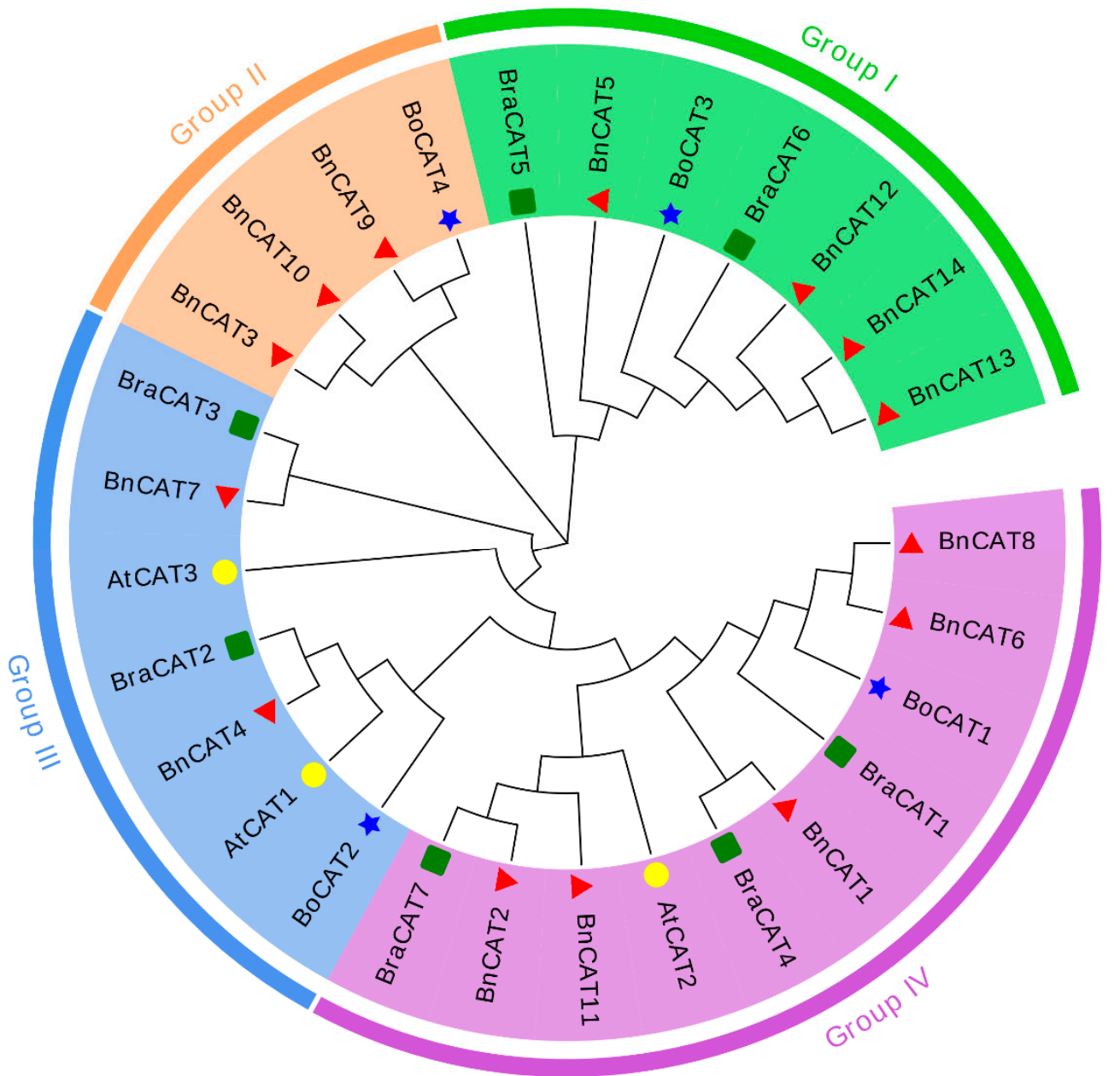

Figure 1. A neighbor-joining phylogenetic tree analysis of CAT proteins in Brassica napus, Brassica oleracea, Brassica rapa, and Arabidopsis thaliana. Overall, 14 BnCATs from Brassica napus (red triangle), 4 BoCATs from Brassica oleracea (blue star), 7 BraCATs from Brassica rapa (green box), and 3 AtCATs from Arabidopsis thaliana (yellow circles) were clustered into four groups (Groups I-IV), represented by different colors.

\subsection{Synteny Analysis of CAT Genes}

Tandem and segmental duplication promote new gene family members' development and plant genome progression. The segmental and tandem duplication actions in the $B n C A T$ gene family were examined to clarify the rapeseed $B n C A T$ gene duplication actions. The chromosomal dispersals of $12 \mathrm{BnCAT}$ genes were evaluated. According to Figure 2, 9 out of the 19 chromosomes possessed BnCAT genes. Briefly, chromosomes A07, A08, and $\mathrm{C} 07$ have two BnCAT genes, whereas chromosomes A01, A03, A06, C03, C05, and 
C08 possessed only one $B n C A T$ gene (Figure 2). Notably, the remaining chromosomes did not contain any BnCAT gene. Regardless of the chromosomes, A01, A07, and C03 have one and two genes, and no tandem repeat paralogous genes were found in these regions. In addition, six paralogous genes were recognized on the $\mathrm{A} 03, \mathrm{~A} 06, \mathrm{~A} 08, \mathrm{C} 05, \mathrm{C} 07$, and $\mathrm{C} 08$ chromosomes (Figure 2). One tandem duplication on chromosome A07, one proximal duplication on chromosome A08, and 6 duplication gene pairs were detected (Figure 2). These outcomes showed that the duplication actions played a crucial role in expanding the $B n C A T$ family genes.

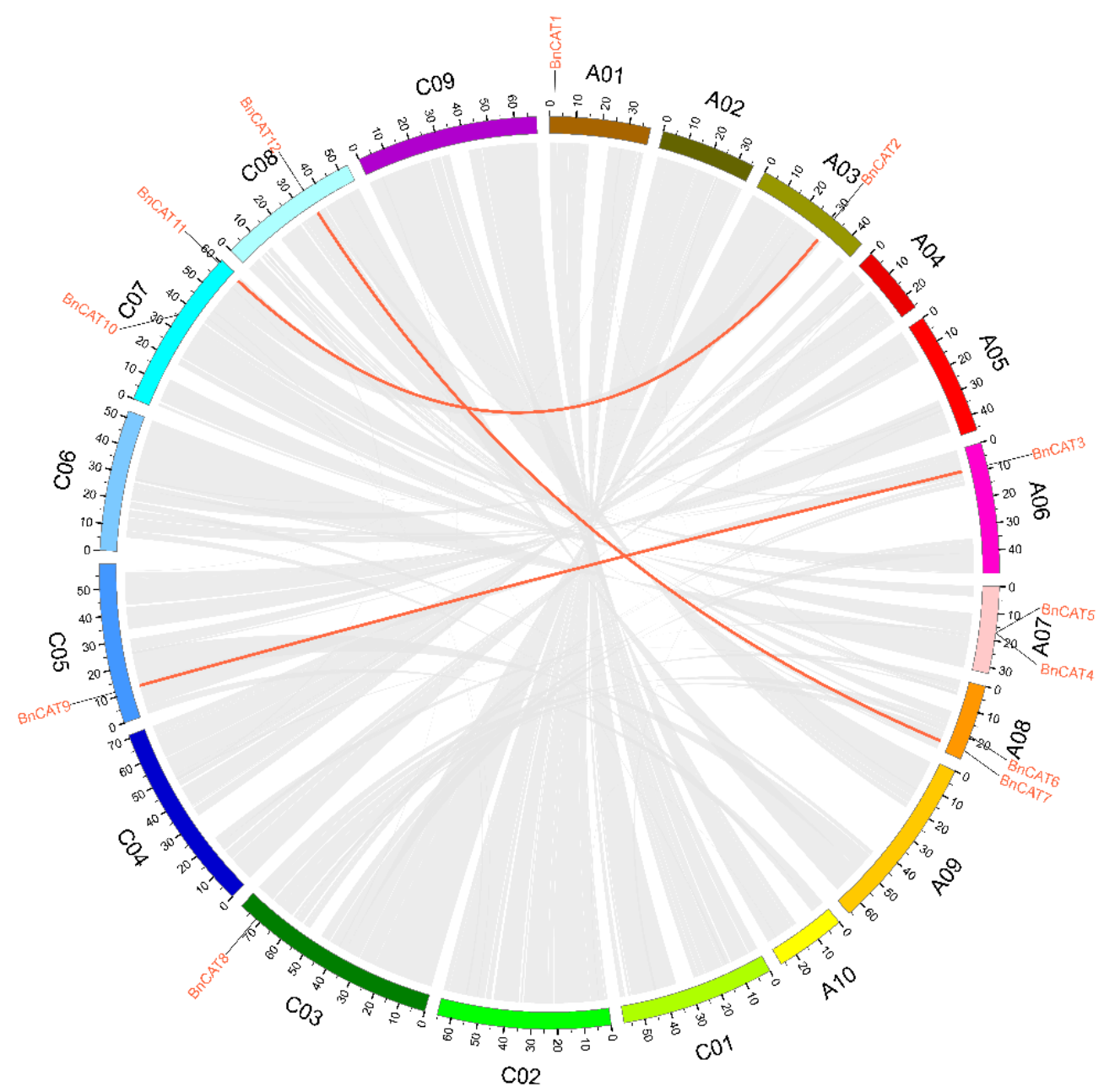

Figure 2. Circular illustrations of the chromosomal dispersal and inter-chromosomal associations of $B n C A T$ genes. Grey lines in the background show all the syntenic blocks in the rapeseed genome, and the red lines show syntenic CAT gene pairs.

Collinearity analysis discovered robust orthologs of the $C A T$ genes among B. napus and the other three inherited plant species (Figure 3; Table S3). Briefly, in the A subgenome, $4 \mathrm{~B}$. napus genes displayed syntenic associations with 3 AtCATs and 1 BraCAT. On the other hand, in the $C$ subgenome, $3 B$. napus genes showed syntenic associations with 2 AtCATs and 1 BoCAT (Figure 3). Notably, several homologous of $A$. thaliana, B. rapa, and $B$. oleracea sustained a syntenic association with $B n C A T s$, suggesting that whole-genome duplication played a key role in BnCATs gene family evolution along with segmental repetition (Table S3). 


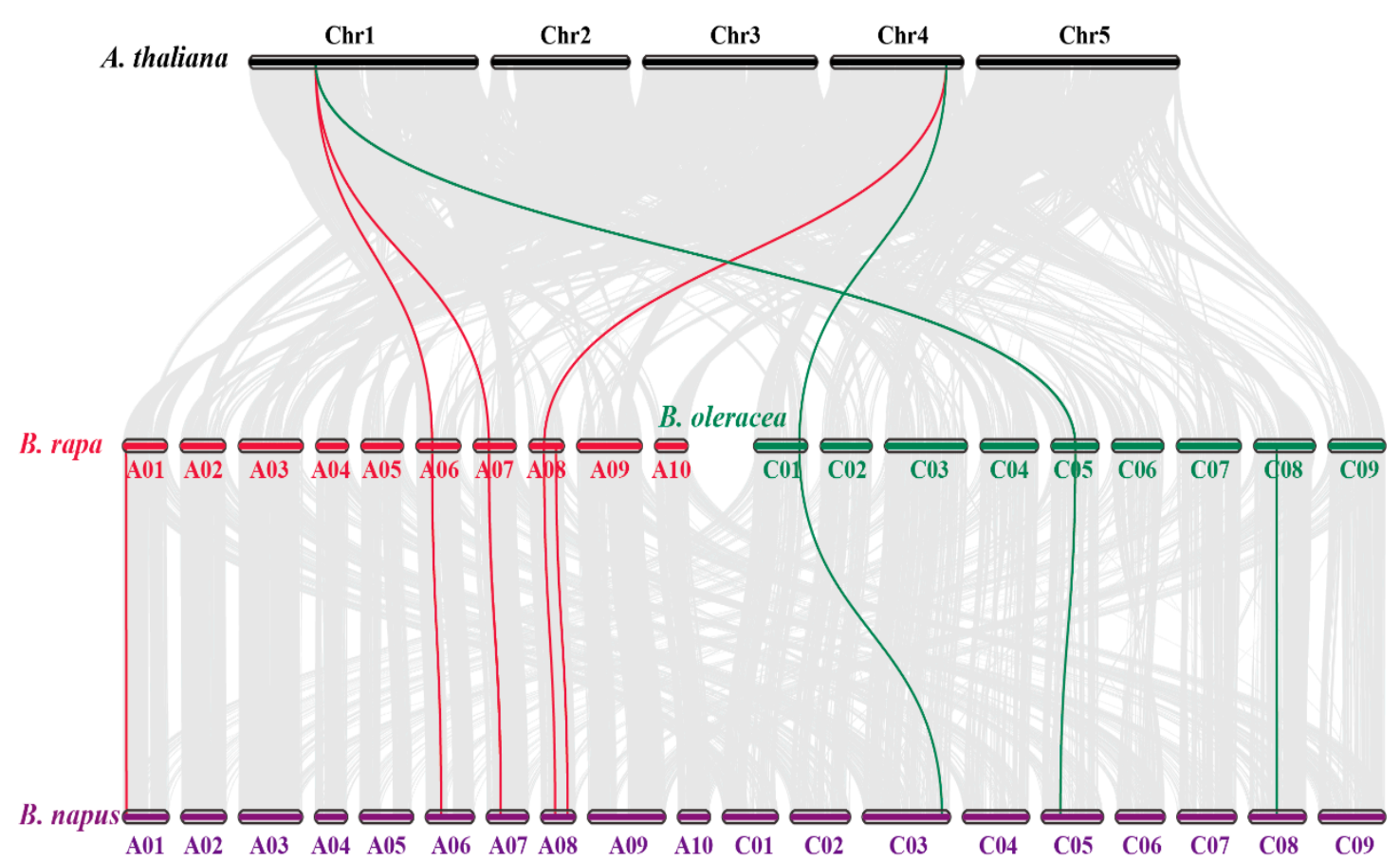

Figure 3. Synteny analysis of CAT genes in B. napus, B. rapa, B. oleracea, and A. thaliana chromosomes. Grey lines in the background show the collinear blocks within rapeseed and other plant genomes, while the red and green lines highlight the syntenic $C A T$ gene pairs. Genes positioned on the B. napus A genome are syntenic with B. rapa and A. thaliana, while genes positioned on the $B$. napus $C$ genome are syntenic with $B$. oleracea and $A$. thaliana.

To comprehend the evolutionary constrictions on the BnCAT gene family, the Ka, Ks, and $\mathrm{Ka} / \mathrm{Ks}$ ratio was assessed for rapeseed. Our findings presented that all the duplicated $B n C A T$ gene pairs had a Ka/Ks ratio of $<1$ (Table S3), demonstrating that the rapeseed CAT family genes might have faced selective pressure, a discriminating burden, throughout its evolution.

\subsection{Analysis of BnCAT Gene Structures and Conserved Motifs}

The exon-intron configurations of the $B n C A T$ genes were observed to boost our understanding of the rapeseed $C A T$ family genes' development. We found that introns of the BnCATs ranged from 6 to 14 (Figure 4A). Groups III and IV contained 6 to 7 introns, Group I includes 7 to 8 introns, whereas Group I had the highest number of introns (11 and 14). Similarly, Groups I, III, and IV contain 4 to 6 exons, while Group I had the highest number of exons (12 and 15). Some of the exons are overlapped with the catalase-domain regions (Table 1; Figure 4A). Briefly, Groups I, III, and IV showed similar intron/exon association patterns, and only Group I had a diverse intron/exon association pattern. These findings specified that members inside a group had remarkably identical gene structures, consistent with their phylogenetic associations.

Moreover, we investigated the full-length protein sequences of 14 BnCATs and 3 AtCATs via MEME software to recognize their conserved motifs. The conserved motif of the $C A T$ genes ranged from 8 to 15 . Overall, 8 conserved motifs were identified, and the motif distributions were also similar within the groups (Groups I-IV) (Figure 4B; Table S4). For instance, Groups I, III, and IV contain 8 conserved motifs, except BnCAT13. Moreover, all members of Group II contained 15 conserved motifs (Figure 4B). In conclusion, the group classifications' consistency was powerfully maintained by analyzing the conserved motifs' composition, gene structures, and phylogenetic relationships, signifying that the CAT proteins have extremely preserved amino acid remains, and members inside a group may have parallel roles. 
A

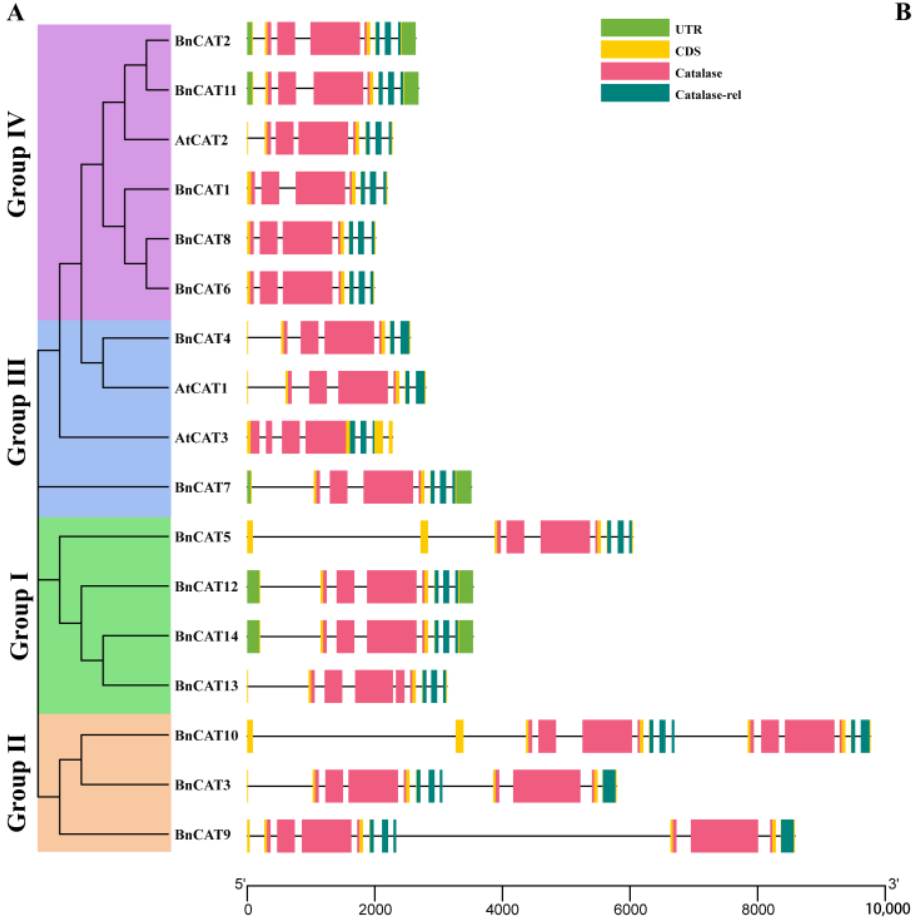

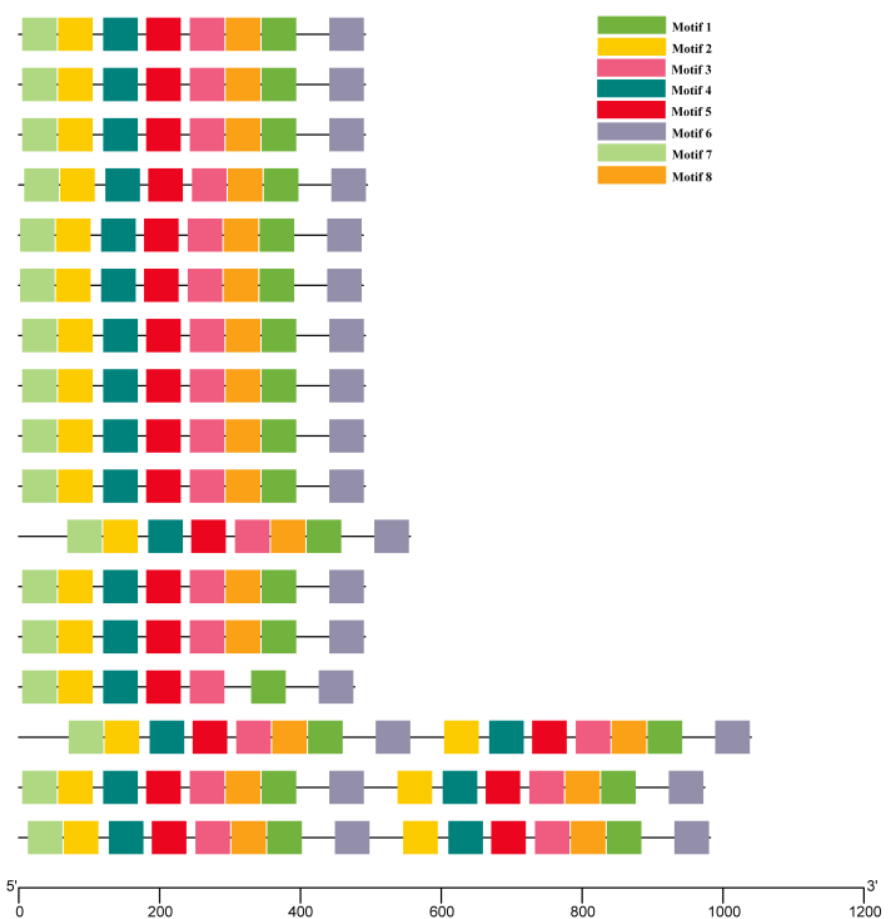

Figure 4. The gene structure and motif analysis of the CAT family genes from B. napus and A. thaliana. According to the phylogenetic relationships, the CAT genes from both genomes were clustered into four groups (Groups I-IV). (A) The gene structure of the BnCATs and AtCATs. Light green color shows the UTR regions, yellow color shows the CDS or exons, black horizontal line shows the introns, pink color shows the catalase core domain (catalase, PF00199), and dark green color shows the catalase-related immune-responsive domain (catalase-rel, PF06628). (B) Conserved domain structures identified in the BnCATs and AtCATs. Different color boxes show different motifs.

\subsection{Cis-Elements in Promoters of BnCAT Genes}

To recognize the gene functions and regulatory patterns, the cis-elements in the promoters of the $B n C A T$ genes were investigated by searching a 2000 base-pair (bp) region from the transcriptional activation site of each gene against the PlantCARE database. As shown in Figure 5, several cis-regulatory elements were predicted in the BnCAT promoter. The detailed information of the identified elements is presented in Table S5. Overall, five different hormone-correlated responsive elements related to abscisic acid (ABA), auxin, methyl jasmonate (MeJA), gibberellin (GA), and salicylic acid (SA) were identified, suggesting that these genes may respond to phytohormones (Figure 5; Table S5). Most of the hormone-correlated responsive elements are very specific to some genes, as shown in Figure 5. Among them, the ABA-, MeJA-, and GA-responsive elements are widely distributed and commonly present in several genes, highlighting their vital roles in phytohormone responses (Figure 5).

Additionally, four different stress-related responsive elements (drought, low-temperature, anaerobic, and light) were also predicted, indicating that these genes may respond to stress stimulates (Figure 5; Table S5). Moreover, a number of light-responsive elements were observed, suggesting the significant role of $B n C A T s$ in response to light stress. Besides, one defense and stress-responsive element were identified in BnCAT1, BnCAT2, BnCAT5, $B n C A T 8$, and BnCAT9 genes (Figure 5; Table S5), demonstrating that these five genes might participate against defense and stress. These hormone and stress-associated elements in the $B n C A T$ promoters suggest that transcript profiling of $B n C A T s$ may vary with hormones and abiotic stresses. 


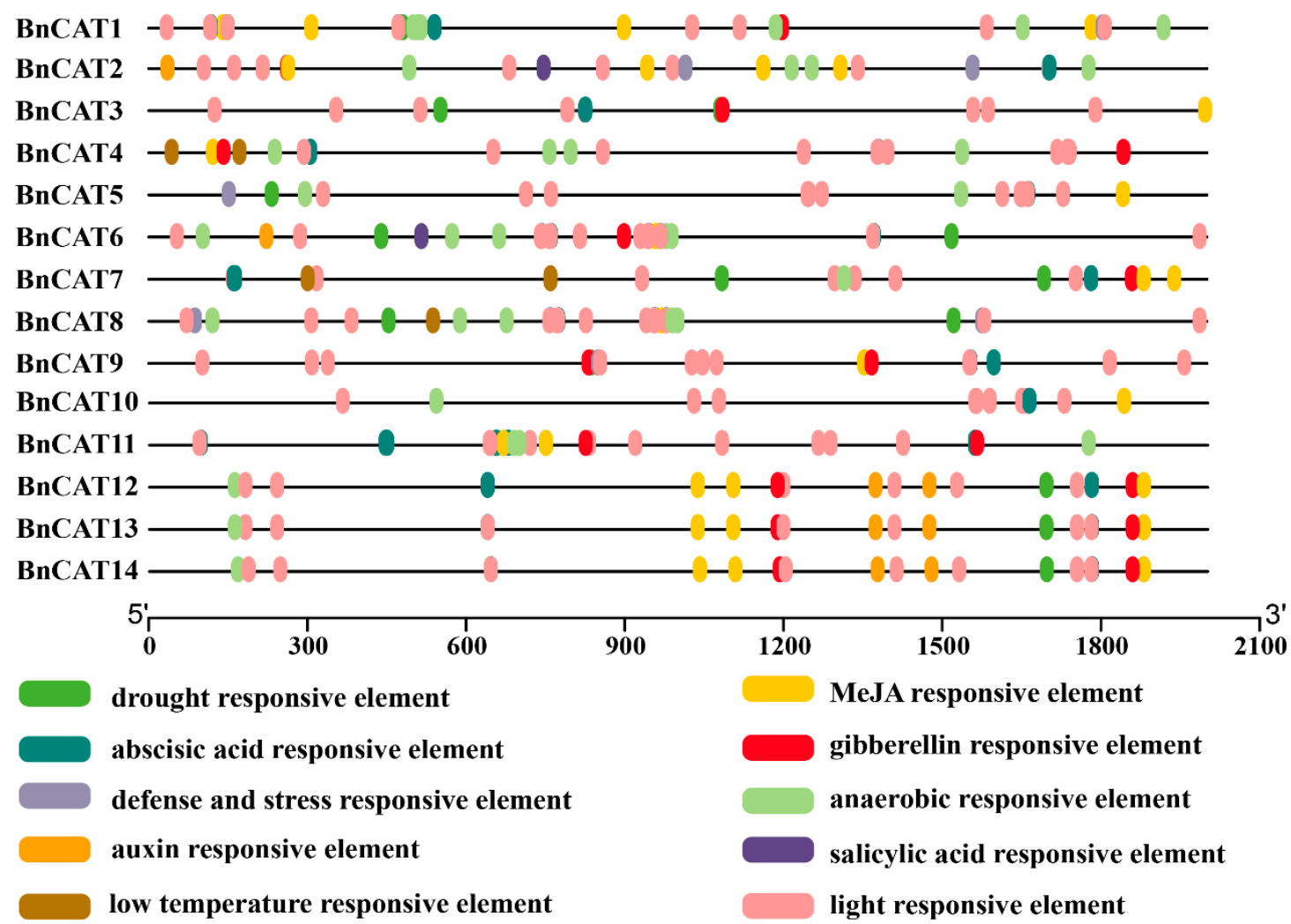

Figure 5. Cis-elements in the promoters of the BnCAT genes that are associated with different hormone- and stress-responsive elements. Different color boxes show different identified cis-elements.

\subsection{Genome-Wide Analysis of miRNA Targeting BnCAT Genes}

Over the past few years, a significant number of investigations have revealed that miRNA-mediated regulation is linked with the stress response in plants. Therefore, to boost our understanding of the miRNAs associated with the regulation of BnCAT genes, we identified 6 putative miRNAs targeting $3 B n C A T$ genes, as shown in the network diagram (Figure 6). The detailed information of the miRNA targeted sites is presented in Table S6. We found that five members of the bna-miR166 family targeted two genes (BnCAT6 and $B n C A T 8$ ), and one member of the bna-miR393 family targeted one gene (BnCAT4) (Figure 6; Table S6). Particularly, BnCAT6 and BnCAT8 were predicted to be targeted by five miRNAs (bna-miR166a, bna-miR166b, bna-miR166c, bna-miR166d, and bna-miR166e).
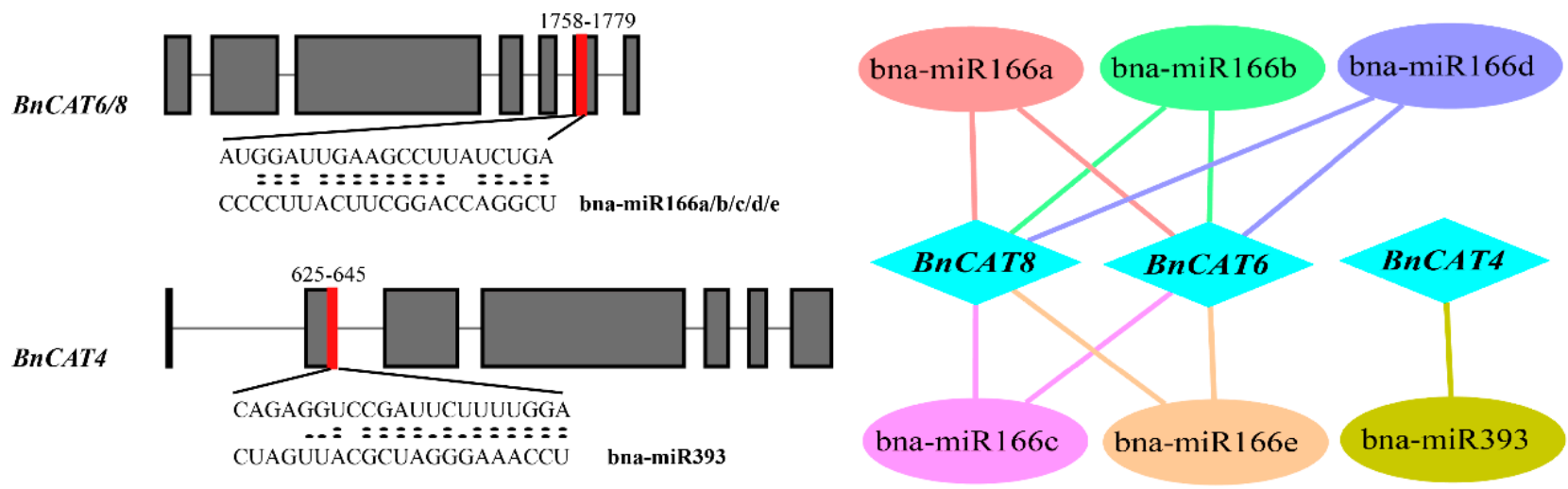

Legend: $\square$ Exon $\square$ UTR Intron I miRNA complementary sites with the positions of BnCAT gDNAs

Figure 6. A network illustration of the regulatory associations among the presumed miRNAs and selective $B n C A T$ genes. 


\subsection{Functional Annotation Analysis of BnCAT Genes}

To further recognize the $B n C A T$ genes' functions, we performed GO annotation and enrichment analysis based on biological process (BP), molecular function (MF), and cellular component (CC) classes; these terms help us understand the function of genes at a molecular level. The annotation results of BP, MF, and CC exhibited several significantly enriched terms (Table S7). Some of the most enriched terms are highlighted in the subsequent section.

The GO-CC enrichment analysis confirmed that 14 terms are highly enriched, such as peroxisome (GO:0005777), cytoplasmic part (GO:0044444), cellular_component (GO:0005575), organelle (GO:0043226), cytoplasm (GO:0005737), mitochondrion (GO:0005739), and cell (GO:0005623), etc. (Table S7). Some of these terms are consistent with the subcellular localization prediction of the CAT proteins. The GO-MF enrichment results detected 13 highly enriched terms, including catalase activity (GO:0004096), organic cyclic compound binding (GO:0097159), oxidoreductase activity, acting on peroxide as acceptor (GO:0016684), catalytic activity (GO:0003824), antioxidant activity (GO:0016209), and peroxidase activity (GO:0004601), etc. (Table S7). These terms also confirm the function of BnCAT genes in ROS scavenging and antioxidant defense systems. Likewise, the GO-BP results predicted a vast number (33) of significantly enriched terms. The most common and useful terms are a cellular response to stimulus (GO:0051716), ROS metabolic process (GO:0072593), response to stress (GO:0006950), response to ROS (GO:0000302), response to hydrogen peroxide (GO:0042542), and cellular detoxification (GO:1990748), etc. (Table S7). Overall, GO enrichment analysis confirms the functional role of $B n C A T$ genes in several cellular, molecular, and biological processes related to ROS, antioxidant enzymes, and response to stresses.

\subsection{Expression Profiling of BnCAT Genes in Various Tissues}

To illustrate the transcript levels of the BnCAT genes, we examined 11 tissues and organs of rapeseed at various growth phases based on RNA-seq data from rapeseed (ZS11 variety) (BioProject ID PRJCA001495). The expression profiles of the BnCAT genes varied in the various tissues and organs. For instance, the expression patterns of most $B n C A T$ genes in stem, leaf, and silique- $5 \mathrm{~d}$, silique-7d, silique-10d, and silique-14d were higher than those of other tissues (Figure 7). The expression levels of BnCAT1 (TPM $=0.00-6.44$ ), BnCAT3 (TPM = 0.00-9.91), and BnCAT9 (TPM = 0.01-3.45) were much lower, respectively; in contrast, $B n C A T 4$ (TPM $=14.98-374.72)$ and $B n C A T 10(\mathrm{TPM}=40.89-287.88)$ were highly expressed in most of the tissues, respectively, suggesting that these candidate genes may play diverse roles in regulating rapeseed growth processes (Figure 7).

\subsection{Expression Profiles of BnCAT Genes under Phytohormones and Abiotic Stress Conditions}

Under abiotic and phytohormone stress conditions, rapeseed growth and development were affected by physiological, biochemical, and molecular levels. Therefore, qRT-PCR was applied to investigate $14 B n C A T$ genes' expression levels at different time points after drought, salinity, cold, waterlogging, ABA, GA, MeJA, and IAA treatments (Figure 8). Under hormone stress conditions, most of the genes showed relatively low expression levels, except for some genes. For instance, BnCAT12 in response to ABA (2.73 and 3.40 fold), GA (3.67 and 3.48 fold), and IAA (8.85 and 6.08 fold), as well as BnCAT13 in response to ABA (2.72 and 3.58 fold), GA (3.24 and 3.04 fold), and IAA (9.47 and 5.61 fold) at 6 and $8 \mathrm{~h}$ were significantly upregulated, respectively. Further, BnCAT10 (1.99-2.08 fold) display substantially higher expression at 6 and $8 \mathrm{~h}$ under ABA treatment, respectively (Figure 8A). Under GA treatment, BnCAT1 (2.48-3.17 fold) at 4 and $6 \mathrm{~h}$, and BnCAT2 (2.71 fold) at $4 \mathrm{~h}$ were expressively upregulated, respectively (Figure $8 \mathrm{~A}$ ). Only BnCAT4 (27.49-34.37 fold) was upregulated after 6 and $8 \mathrm{~h}$ of the MeJA treatment, respectively (Figure 8A). However, BnCAT5-BnCAT10 and BnCAT14 genes did not display any significant difference throughout the hormone treatments (Figure $8 \mathrm{~A}$ ). 


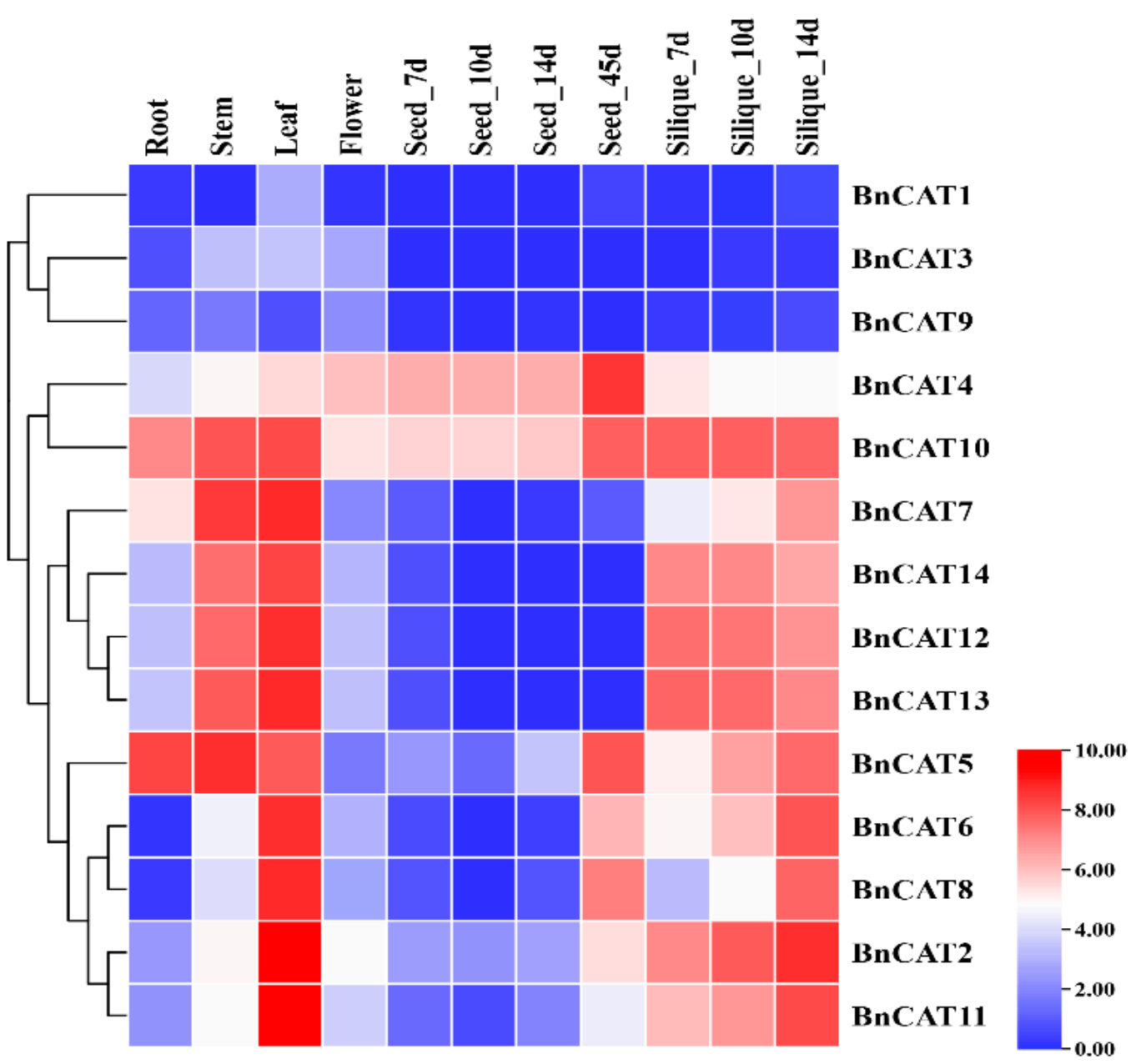

Figure 7. Expression patterns of $B n C A T$ genes in various tissues at different growth phases of rapeseed. The $7 d, 10 d, 14 d$, and $45 \mathrm{~d}$ labels showed the time-points when the samples were harvested. In the expression bar, the red color shows high, and the blue color shows low expression levels.

Meanwhile, a relatively high number of $B n C A T$ genes were upregulated by abiotic stresses compared to hormone treatments concerning their control conditions (Figure $8 \mathrm{~B}$ ). Specifically, BnCAT1-BnCAT3 (1.33-1.41 fold) and BnCAT11-BnCAT13 (1.36-1.97 fold) were upregulated by cold stress at the early or late response, respectively (Figure $8 \mathrm{~B}$ ). Under salinity stress conditions, BnCAT1-BnCAT3 (1.75-2.03 fold), BnCAT11 (1.46-1.84 fold), and $B n C A T 12$ (1.25-2.21 fold) were upregulated at different time points of the treatment, respectively. Likewise, only BnCAT1 (31.95 fold) was induced by drought stress at $2 \mathrm{~h}$, and the other genes were not affected by the drought stress, respectively (Figure 8B). Interestingly, almost all of the genes were significantly upregulated at all time points under waterlogging stress, except BnCAT8-BnCAT10 (0.14-0.87 fold), respectively, which showed relatively low expressions (Figure 8B). Briefly, some genes, such as BnCAT1, BnCAT2, BnCAT3,BnCAT11, $B n C A T 12$, and $B n C A T 13$, showed a similar expression pattern during ABA (0.93-1.33, $0.60-1.15,0.56-1.47,0.91-1.34,0.69-3.40$, and 0.75-3.58 fold), GA (1.47-3.17, 1.13-2.70, $0.99-2.14,0.73-1.45,1.63-3.67$, and 1.55-3.24 fold), IAA (0.98-2.58, 1.30-2.12, 0.85-2.15, $2.76-5.02,3.72-8.85$, and 3.97-9.47 fold), cold (0.97-1.34, 1.12-1.41, 1.09-1.33, 1.16-1.71, 1.44-1.98, and 1.11-1.89 fold), and salinity (1.11-2.30, 1.30-1.75, 1.22-2.11, 1.47-1.84, 0.98-2.21, and $0.77-1.70$ fold) treatments, respectively (Figure 8 ). The above outcomes suggest that these genes may be crucial for enlightening resistance to multiple stresses, especially waterlogging stress. 
(A)

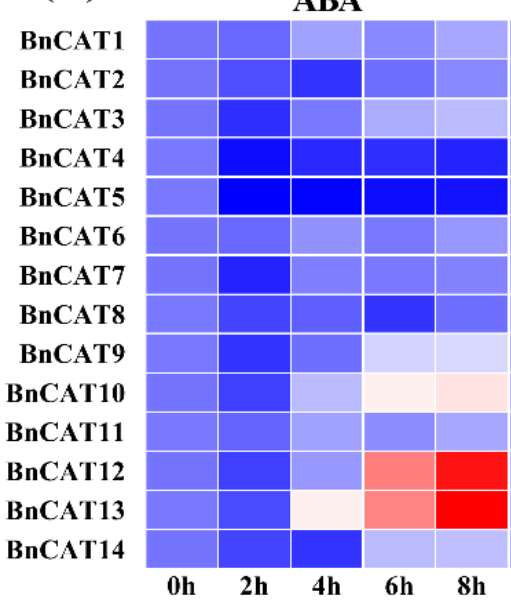

GA

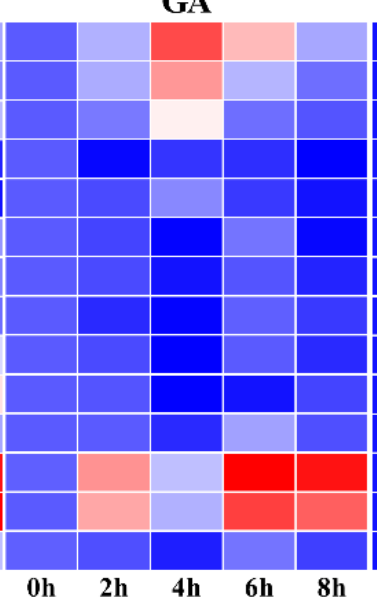

IAA

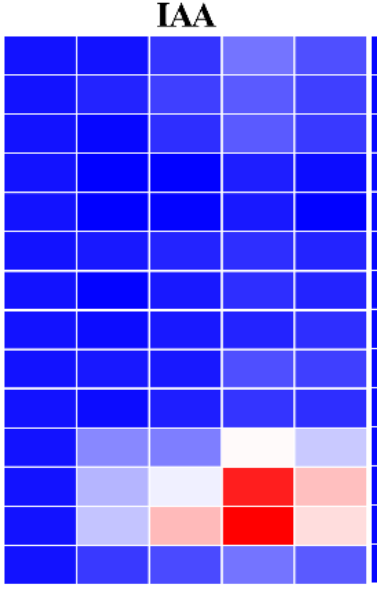

MeJA

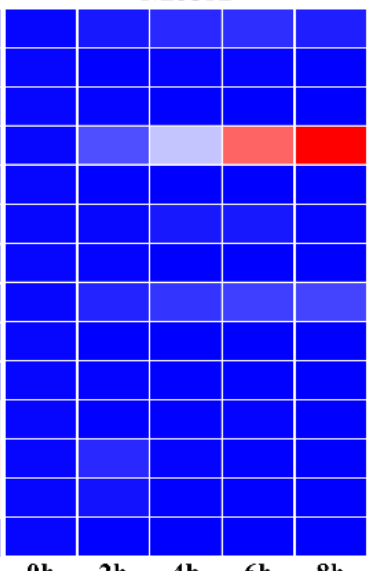

PEG

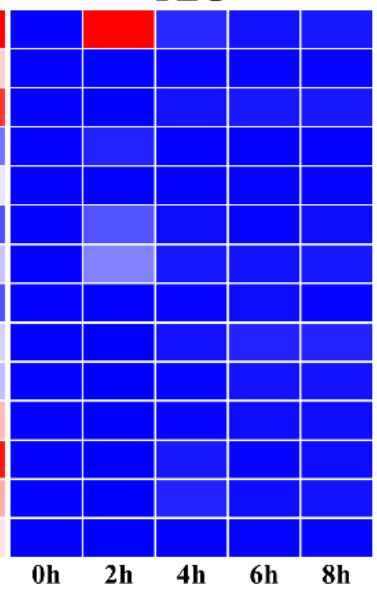

WL

30
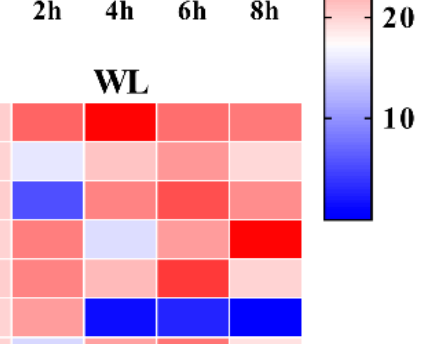

BnCAT1
BnCAT2
BnCAT3
BnCAT4
BnCAT5
BnCAT6
BnCAT7
BnCAT8
BnCAT9
BnCAT10
BnCAT11
BnCAT12
BnCAT13
BnCAT14

BnCAT1
BnCAT2
BnCAT3
BnCAT4
BnCAT5
BnCAT6
BnCAT7
BnCAT8
BnCAT9
BnCAT10
BnCAT11
BnCAT12
BnCAT13
BnCAT14

BnCAT1
BnCAT2
BnCAT3
BnCAT4
BnCAT5
BnCAT6
BnCAT7
BnCAT8
BnCAT9
BnCAT10
BnCAT11
BnCAT12
BnCAT13
BnCAT14

BnCAT1
BnCAT2
BnCAT3
BnCAT4
BnCAT5
BnCAT6
BnCAT7
BnCAT8
BnCAT9
BnCAT10
BnCAT11
BnCAT12
BnCAT13
BnCAT14

BnCAT1
BnCAT2
BnCAT3
BnCAT4
BnCAT5
BnCAT6
BnCAT7
BnCAT8
BnCAT9
BnCAT10
BnCAT11
BnCAT12
BnCAT13
BnCAT14

BnCAT1
BnCAT2
BnCAT3
BnCAT4
BnCAT5
BnCAT6
BnCAT7
BnCAT8
BnCAT9
BnCAT10
BnCAT11
BnCAT12
BnCAT13
BnCAT14

BnCAT1
BnCAT2
BnCAT3
BnCAT4
BnCAT5
BnCAT6
BnCAT7
BnCAT8
BnCAT9
BnCAT10
BnCAT11
BnCAT12
BnCAT13
BnCAT14

BnCAT1
BnCAT2
BnCAT3
BnCAT4
BnCAT5
BnCAT6
BnCAT7
BnCAT8
BnCAT9
BnCAT10
BnCAT11
BnCAT12
BnCAT13
BnCAT14

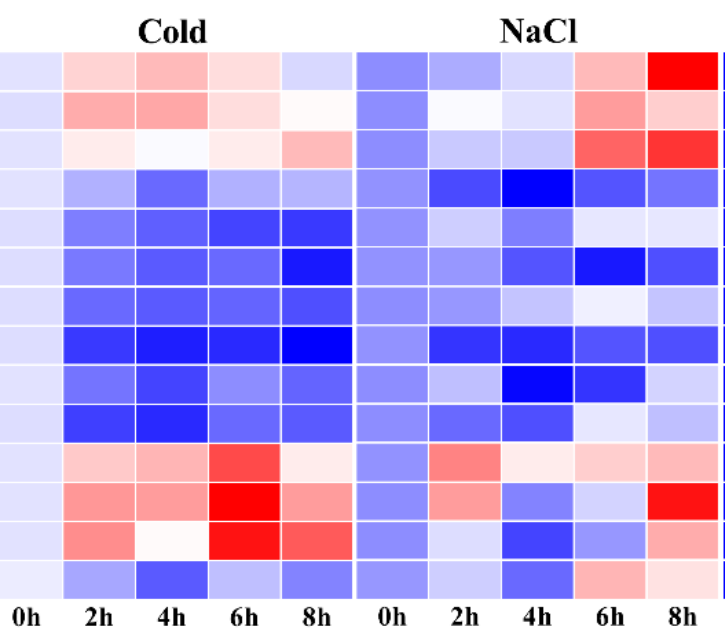

$\mathrm{NaCl}$

Figure 8. Expression patterns of the $B n C A T$ genes under various hormones and abiotic stress. (A) Expression profiling during hormone treatments, including abscisic acid (ABA), gibberellic acid (GA), indole-acetic acid/auxin (IAA), and methyl jasmonate (MeJA). (B) Expression profiling under abiotic stresses, including cold, salinity (NaCl), drought (PEG-6000), and waterlogging (WL). The $0 \mathrm{~h}(\mathrm{CK}), 2 \mathrm{~h}, 4 \mathrm{~h}, 6 \mathrm{~h}$, and $8 \mathrm{~h}$ labels displayed the time points (hours) when the samples were harvested for expression analysis after the stress treatment. The bars show the comparative gene expression patterns examined via the $2^{-\Delta \Delta C T}$ method. The red color shows high, and the blue color shows low expression levels.

\section{Discussion}

\subsection{Characterization of CAT Gene Family in Rapeseed}

Rapeseed is an allotetraploid type that practiced extensive genome doubling and integration procedures [40]. In the current study, we recognized $14 B n C A T$ genes via genome-wide analysis that surpasses the 4 BoCATs and 7 BraCATs (Table 1; Table S2). Generally, the $C A T$ gene family of plants usually consists of a small number of genes. Each CAT member in A. thaliana was naturally homologous to 2-3 genes in the rapeseed genome. These results are supported by the previous discovery that $1 A$. thaliana gene paralleled to 2 or $>2$ homologous genes in rapeseed [41]. According to the phylogenetic investigation, $14 \mathrm{BnCATs}$ were classified into four groups. CAT genes from $A$. thaliana were classified into the Group III and Group IV together with some BnCATs genes, BoCATs, and BraCATs (Figure 1), signifying comparable evolutionary lines among B. napus, B. oleracea, B. rapa, and A. thaliana. These alliances in the phylogenetic tree was further confirmed by the gene structure analysis (Figure 4). The locations and numbers of introns of the CAT genes were found to be extremely conserved between 13 angiosperm plants (monocots and dicots). This result suggests that one ancestral duplicate of the CAT gene, comprising seven introns, have gone over numerous rounds of intron loss and gain throughout evolution [42]. Moreover, the 
motif patterns were also comparable within the group classification (Figure 4B). Notably, Group II contains diverse motif patterns, suggesting that BnCATs possess exceptionally conserved protein structures (Figure 4B). These results are in agreement with previous findings of cotton, where genes within the same group contain diverse gene structures and motifs patterns [18].

\subsection{The Critical Role of CAT Genes in ROS Metabolism, Hormone, and Abiotic Stress Responses}

Catalase, a robust enzyme that facilitates the ROS-scavenging procedure, serves as a crucial player in plant growth, development, and abiotic stress response [2,3]. To date, it has been extensively studied in several plant species against various stresses, such as A. thaliana [43-47], rice [48-50], wheat [51-53], sugarcane [21,54,55], cucumber [16,56,57], cotton $[18,58,59]$, and sweet potato $[20,60,61]$.

Under drought and salinity stress, the AtCAT1 transcript level was triggered by a MAPK kinase (AtMEK1), which was associated with ROS generation (mainly $\mathrm{H}_{2} \mathrm{O}_{2}$ ) [62]. The transcription factor WRKY75 suppressed the expression level of AtCAT2 to improve the ROS accretion, leading to leaf senescence in A. thaliana [63]. In another study, the transcript level of $A t C A T 2$ was repressed to activate endogenous ROS generation under lead toxicity [64]. Further, Due et al. [14] discovered that AtCAT1 played a significant role in eliminating abiotic stress-persuaded $\mathrm{H}_{2} \mathrm{O}_{2}$, whereas AtCAT2 and AtCAT3 ensured $\mathrm{H}_{2} \mathrm{O}_{2}$ scavenging under light and dark conditions, respectively. Additionally, AtCAT3 was phosphorylated through a calcium-associated protein kinase to intercede $\mathrm{H}_{2} \mathrm{O}_{2}$ homeostasis inside the peroxisomes of guard cells under drought conditions, and the over-expression of AtCAT3 improved the drought resistance in A. thaliana [43].

In rice, three $C A T$ genes (OsCat $A, O s C a t B$, and $O s C a t C$ ) were triggered under different stress conditions; further, these genes were also involved in ROS homeostasis, root evolution stimulation, and photorespiration [15]. The over-expression of OsCatA and OsCatC improved the drought tolerance in transgenic rice [15]. In another study, Vighi et al. [65] reported that $O s C a t A$ and $O s C a t B$ were strongly induced by high salinity and cold stress in a tolerant genotype compared with that in a sensitive genotype. In cucumber, the expression level of CsCAT1 was triggered by ABA treatment, whereas CsCAT2 was repressed under drought stress and CsCAT3 upregulated after salinity, drought, and ABA treatment [16]. In sugarcane, the expression pattern of $S c C A T 1$ was induced in response to oxidative, salinity, heavy metal, and drought stresses [55]. In sweet potato, the transcript level of IbCAT2 was stimulated under drought and salinity conditions, and the over-expression of this gene increases the tolerance to both stresses in Saccharomyces cerevisiae and E. coli [20].

In this study, BnCAT1, BnCAT2, BnCAT3, BnCAT12, and BnCAT13 were mainly induced by ABA, GA, IAA, cold, and salinity. Interestingly, almost all identified genes were strongly influenced by waterlogging treatment, except $B n C A T 8-B n C A T 10$, which showed relatively low expressions throughout the stress; this indicates that these genes play a major role in waterlogging stress tolerance in rapeseed. Overall, these findings suggest that $C A T$ genes play a significant part in activating ROS metabolism and respond to numerous abiotic cues (Figure 8). These results were also confirmed by the GO enrichment analysis, where most of the GO terms were found to be associated with ROS metabolism and abiotic stresses (Table S4). In future work, stress-related CAT genes can be used for functional validation in rapeseed to get further insight into their actual stress tolerance mechanisms.

\subsection{Expression Pattern of CAT Genes in Various Tissues}

The expression patterns of the CAT genes in different tissues have been described for numerous plant species. For example, in rice, OsCATA displayed a relatively higher expression level at all the developmental phases; OsCATC presented a nearly comparable expression pattern as OsCATA, apart from the flowering phase. OsCATD sustained a low constitutive expression level during the complete growth life cycle of rice [17]. In cotton, GhCAT1-GhCAT4 significantly showed higher expression in almost all the growth and developmental stages (root, stem, leaf, petal, calycle, torus, stamen, pistil, and fiber devel- 
opment), suggesting that the CAT gene family is possibly associated with the growth and development of various tissues in cotton [18]. In cucumber, CsCAT1 and CsCAT2 showed higher transcript levels in leaves and considerable expression in fruit and roots, whereas CsCAT2 showed a stronger transcript pattern in fruits, flowers, stem, and leaves [16]. In the current study, the results showed that many genes showed higher transcript levels in the root, stem, leaf, and silique (Figure 7). Only BnCAT4 (TPM = 82.23-374.72) and BnCAT10 (TPM = 49.85-219.51) exhibited higher expression throughout the seed expansion stages. The expression levels of 3 genes $(B n C A T 1$ (TPM $=0.00-6.44)$, BnCAT3 $(\mathrm{TPM}=$ $0.00-9.91)$, and BnCAT9 (TPM = 0.01-3.45) in all the tissues were almost undetectable in rapeseed, indicating that these genes might do not play any role in rapeseed growth and development (Figure 7).

\section{4. miRNA: Key Players in the Regulation of Stress Responses}

MicroRNAs (miRNAs), a group of single-stranded, non-coding micro RNAs, have been revealed to be involved in post-transcriptional gene regulation $[30,66]$. During the past few years, numerous miRNAs have been identified via genome-wide analysis in rapeseed that responds to different environmental stresses [67-73].

The current study recognized five members of the bna-miR166 family and one member of the bna-miR393 family targeting three CAT genes (BnCAT4, BnCAT6, and BnCAT8) (Figure 6). miR166 has been reported to be significantly upregulated in response to UV-B radiation in maize [74]; in cassava under cold and drought stresses conditions [75]; and in Chinese cabbage under heat stress conditions [75]. Likewise, miR393 has also been reported to be strongly linked with stress responses, such as drought and salinity in French bean (Phaseolus vulgaris) [76], cold stress in tea plant (Camellia sinensis) [77], and nitrogen deficiency in maize [78]. Some of these miRNAs have also been reported in rapeseed, playing a significant role in rapeseed genetic improvement [67-73]. These studies suggest that these bna-miRNAs might play decisive roles against numerous stresses by modifying the transcript level of the CAT genes in rapeseed.

\section{Materials and Methods}

\subsection{Identification of CAT Family Genes}

In this work, two approaches were used to identify the CAT genes in rapeseed. Firstly, the rapeseed genome (ZS11 genotype) sequence was downloaded from the BnPIR database (http:/ / cbi.hzau.edu.cn/bnapus/index.php, accessed on 10 February 2021), and a local rapeseed dataset was used by software blast-2.7.1+ (ftp:/ /ftp.ncbi.nlm.nih. gov/blast/executables/blast+/2.7.1/, accessed on 10 February 2021). The amino acid sequences of 3 A. thaliana CAT family genes (AtCAT1/At1g20630, AtCAT2/At4g35090, and AtCAT3/At1g20620) were obtained from the TAIR Arabidopsis genome database (http://www.arabidopsis.org/, accessed on 10 February 2021). These sequences were used as a reference to identify the CAT genes in the local rapeseed database.

Secondly, the HMMER 3.1 (http: / / www.hmmer.org/, accessed on 10 February 2021) and BLASTP with the threshold e-value set to $1 \mathrm{e}^{-5}$ were performed using the Hidden Markov Model (HMM V 3.0) profiles of the catalase (PF00199) and the catalase-related immune-responsive domain (PF06628) were downloaded from the Pfam protein database (http://pfam.xfam.org/, accessed on 10 February 2021) and used as the inquiry. The default limitation of HMMER 3.1 was set to 0.01 . We combined all hits based on HMMER and BLASTP results and omitted redundant hits with a similar scaffold or chromosome location due to the rapeseed genome's complexity. The remaining CAT sequences were scrutinized for a catalase-related immune-responsive domain (PF06628) by the Pfam server to confirm that all the identified genes belonged to the CAT gene family. Finally, 14 BnCAT genes were identified in the rapeseed genome. The genomic and protein sequences of all the identified $B n C A T$ genes were obtained from the BnPIR rapeseed database. Moreover, to disclose the CAT genes' evolutionary association in different plant species, the protein sequences of the Brassica rapa and Brassica oleracea CATs were downloaded from the JGI 
Phytozome 12.0 (https:/ / phytozome.jgi.doe.gov/pz/portal.html, accessed on 10 February 2021) database via the same method as described above.

\subsection{Characterization of CAT Family Genes}

The physical and chemical properties, such as the number of amino acids in a sequence, molecular weight (MW), and isoelectric points (pI), were measured using the PROTPARAM tool on the ExPASy website (http:/ / web.expasy.org/protparam/, accessed on 10 February 2021). The subcellular localization of BnCAT proteins was predicted using the WoLF PSORT server (https:/ / wolfpsort.hgc.jp/, accessed on 10 February 2021). The structure of the $B n C A T$ genes was predicted and constructed via TBtools software (V 1.068; https: / / github. com/CJ-Chen/TBtools, accessed on 10 February 2021). Potentially conserved motifs were identified using MEME (V 4.11.4) by analyzing the sequences of 13 BnCAT proteins.

\subsection{Phylogenetic Tree and Synteny Analysis of BnCAT Family Proteins}

To observe the evolutionary past of the rapeseed CAT gene family, we used Brassica napus, Brassica oleracea, Brassica rapa, and A. thaliana protein sequences to create a phylogenetic tree. The protein sequences were multiple-aligned using MEGA X (V 6.06) software. The tree was constructed based on the neighbor-joining (NJ) method with 1000 bootstrap replicates. For better visualization, the tree was observed and modified via the online website Evolview v3 (https:/ / www.evolgenius.info/evolview, accessed on 10 February 2021). Synteny relationships of CAT genes from Brassica napus, Brassica oleracea, Brassica rapa, and Arabidopsis thaliana were developed from JCVI (https:/ / github.com/tanghaibao/jcvi, accessed on 10 February 2021). To investigate the evolutionary constrictions of each CAT gene pairs, the synonymous (Ks), non-synonymous $(\mathrm{Ka})$ substitution, and $\mathrm{Ka} / \mathrm{Ks}$ ratios were calculated using KaKs_Calculator 2.0 (https:/ / sourceforge.net/projects/kakscalculator2/, accessed on 10 February 2021).

\subsection{Cis-Elements Analysis in the BnCAT Gene Promoters}

To classify the putative cis-elements, the 2000 base pairs (bp) upstream of the start codons were downloaded from the rapeseed genome database (BnPIR) for promoter analysis. Then, each promotor's cis-elements were prophesied using the PlantCARE webtool (http:/ /bioinformatics.psb.ugent.be/webtools/plantcare/html/, accessed on 10 February 2021) and were presented using TBtools (V 1.068).

\subsection{Prediction of Putative miRNA Targeting BnCAT Genes and Functional Annotation Analysis}

The gene sequences of the BnCATs were acquiesced as the candidate genes to identify possible miRNAs via observing against the existing rapeseed reference of miRNA sequences via the psRNATarget database (http:/ / plantgrn.noble.org/psRNATarget/, accessed on 10 February 2021) with default parameters. Cytoscape (V3.8.2, https: / / cytoscape. org/download.html, accessed on 10 February 2021) software was used to create the interaction network between the prophesied miRNAs and the equivalent target $B n C A T$ genes.

To predict the functional annotation of $B n C A T$ genes, we performed the gene ontology (GO) annotation analysis by submitting all $B n C A T$ gene sequences to the eggNOG database (http:/ / eggnog-mapper.embl.de/, accessed on 10 February 2021). Then, the GO annotation data was handled in TBtools for GO enrichment analysis.

\subsection{Expression Analysis of BnCAT Genes in Different Tissues}

The temporal and spatial expression patterns of the candidate BnCAT gene family were evaluated using the RNA-seq data (BioProject ID: PRJCA001495). We examined the total RNA-seq data of the root, stem, leaf, flower, seed, and siliques at the germination stages of the rapeseed variety "ZS11". Raw reads were quality controlled and filtered by software fastp (https:/ / github.com/OpenGene/fastp, accessed on 10 February 2021), and then mapped to the reference genome with software HISAT2 (https: / / github.com/infphilo/hisat2, 
accessed on 10 February 2021). Sequence alignment map (SAM) files were converted to binary alignment map (BAM) and sorted with software Samtools (http: / / www.htslib.org/, accessed on 10 February 2021). Cuffquant and Cuffnorm were used to generate normalized counts in transcripts per million (TPM) values. Based on TPM values, the expression heat map was created using GraphPad Prism 9.0.0 software (https:/ / www.graphpad.com/, accessed on 10 February 2021).

\subsection{Plant Material and Stress Conditions}

In this study, the rapeseed genotype "ZS11", a typical cultivated variety, was used for stress treatments. The seeds of ZS11 genotype was furnished by OCRI, CAAS, China. Before stress treatments, some seeds were randomly selected from the same batch of seeds to determine the germination rate. The seeds with a $100 \%$ germination rate were considered vigorous seeds. The vigorous seeds were carefully chosen and sterilized with $10 \%$ hypochlorous acid solution for $5 \mathrm{~min}$. The seeds were grown on water-saturated filter paper in a chamber $\left(25^{\circ} \mathrm{C}\right.$ day /night and $16 \mathrm{~h} / 8 \mathrm{~h}$ light/dark cycle $)$ until the radicle's extent reached about $5 \mathrm{~mm}$. For stress treatment, germinated seeds were exposed to $150 \mathrm{mM} \mathrm{NaCl}$ solution for salinity stress, 15\% PEG6000 solution for drought stress, and $4{ }^{\circ} \mathrm{C}$ for cold stress on water-saturated filter paper. To analyze the effect of different phytohormones, the germinated seeds were cultivated in Murashige and Skoog (MS) medium provided with $100 \mu \mathrm{M}$ abscisic acid (ABA), $100 \mu \mathrm{M}$ gibberellic acid (GA), $100 \mu \mathrm{M}$ methyl jasmonate (MeJA), and $100 \mu \mathrm{M}$ indole-acetic acid/auxin (IAA). The samples were collected at 0 (CK), $2,4,6$, and $8 \mathrm{~h}$ after the treatments. Three biological replications were carried out for all the treatments. All the samples were instantly frozen in liquid nitrogen and were stored at $-80{ }^{\circ} \mathrm{C}$ for the next analysis.

\subsection{RNA Extraction and qRT-PCR Analysis}

Total RNA was extracted using TransZol Up Plus RNA Kit (TransGen Biotech, Beijing, China) according to the manufacturer's instructions and was then quantified with a Nanodrop ND-1000 spectrophotometer (Thermo Fisher Scientific, Worcester, MA, USA). RNA was purified using TransScript One-Step gDNA Removal. The first-strand of complementary DNA (cDNA) was synthesized using a cDNA Synthesis SuperMix kit (TransGen Biotech, China), and the cDNA solution was then diluted (20x) with distilled deionized water. The quantitative real-time polymerase chain reaction (qRT-PCR) was carried out with an ABI StepOne real-time fluorescence quantitative PCR instrument (Applied Biosystems, Foster City, CA, USA) using SYBR ${ }^{\circledR}$ Green Supermix (Bio-Rad). The BnACTIN was used as an internal control. The qRT-PCR reaction was performed as follows: $94{ }^{\circ} \mathrm{C}$ for $10 \mathrm{~min}$, followed by 40 cycles of $94^{\circ} \mathrm{C}$ for $15 \mathrm{~s}, 60{ }^{\circ} \mathrm{C}$ for $30 \mathrm{~s}$, and $72{ }^{\circ} \mathrm{C}$ for $10 \mathrm{~s}$. Each qRT-PCR reaction was carried out with three biological triplicates, and the data were examined using the $2^{-\Delta \Delta C T}$ method as described by Wang et al. [79]. All the primers used in this experiment are listed in Table S1. The heat map was created using GraphPad Prism 9.0.0 software.

\section{Conclusions}

This study conducted a genome-wide comprehensive study of the CAT gene family in rapeseed and identified $14 B n C A T$ genes, which were clustered into four groups (Group I-Group IV). To get further insights, gene structure, conserved motifs, cis-elements, GO annotation, and miRNA prediction analysis have also been performed. Additionally, several genes' expression levels were highly expressed in the root, leaf, stem, silique, and late stages of seed development. Likewise, several genes were mainly upregulated in response to the ABA, GA, IAA, cold, drought, and waterlogging treatments. Some of the genes are expressed at specific tissues and stress conditions. Briefly, the wide-ranging information collected in the current study can be exploited for future functional analysis of the $B n C A T$ genes regarding rapeseed growth, development, response to hormones, and abiotic stresses. 
Supplementary Materials: The following are available online at https://www.mdpi.com/article/10 $.3390 /$ ijms22084281/s1, Table S1: The information of primer used in this study for gene expression analysis in qRT-PCR, Table S2: The CAT family genes in Arabidopsis thaliana, Brassica oleracea, and Brassica rapa, Table S3: The information of gene duplication type, $\mathrm{Ka}, \mathrm{Ks}$, and $\mathrm{Ka} / \mathrm{Ks}$ ratio values of B. napus and other three plant species, Table S4: The information of identified 8 motifs in BnCAT proteins, Table S5: Information of hormone- and stress-related cis-elements detected in the promoters regions of $B n C A T s$, Table S6: The information of miRNA targeted BnCAT genes, Table S7: The GO enrichment analysis of $14 \mathrm{BnCAT}$ genes.

Author Contributions: Conceptualization, A.R. and W.S.; methodology, A.R. and W.S.; software, A.R. and W.S.; formal analysis, A.R., W.S., A.G., M.A.H., S.S.M., and W.N.; writing-original draft preparation, A.R., W.S., A.G., M.A.H., S.S.M., and W.N.; writing-review and editing, A.R., W.S., Y.L., and X.Z. (Xiling Zou); supervision, X.Z. (Xuekun Zhang), L.Y., and X.Z. (Xiling Zou); funding acquisition, Y.L. and X.Z (Xiling Zou). All authors have read and agreed to the published version of the manuscript.

Funding: This work was supported by the Science and Technology Innovation Project of the Chinese Academy of Agricultural Sciences.

Institutional Review Board Statement: Not applicable.

Informed Consent Statement: Not applicable.

Data Availability Statement: The datasets used and/or analyzed during the current study are available from the corresponding author on reasonable request. However, most of the data is shown in Supplementary files.

Acknowledgments: The authors are grateful to all lab members for their useful suggestions, support, and encouragement.

Conflicts of Interest: The authors declare no conflict of interest.

\section{References}

1. Raza, A.; Razzaq, A.; Mehmood, S.S.; Zou, X.; Zhang, X.; Lv, Y.; Xu, J. Impact of climate change on crops adaptation and strategies to tackle its outcome: A review. Plants 2019, 8, 34. [CrossRef]

2. Hasanuzzaman, M.; Bhuyan, M.; Zulfiqar, F.; Raza, A.; Mohsin, S.M.; Mahmud, J.A.; Fujita, M.; Fotopoulos, V. Reactive Oxygen Species and Antioxidant Defense in Plants under Abiotic Stress: Revisiting the Crucial Role of a Universal Defense Regulator. Antioxidants 2020, 9, 681. [CrossRef] [PubMed]

3. Mittler, R. ROS are good. Trends Plant. Sci. 2017, 22, 11-19. [CrossRef]

4. Mhamdi, A.; Queval, G.; Chaouch, S.; Vanderauwera, S.; Van Breusegem, F.; Noctor, G. Catalase function in plants: A focus on Arabidopsis mutants as stress-mimic models. J. Exp. Bot. 2010, 61, 4197-4220. [CrossRef]

5. Anjum, N.A.; Sharma, P.; Gill, S.S.; Hasanuzzaman, M.; Khan, E.A.; Kachhap, K.; Mohamed, A.A.; Thangavel, P.; Devi, G.D.; Vasudhevan, P. Catalase and ascorbate peroxidase-Representative $\mathrm{H}_{2} \mathrm{O}_{2}$-detoxifying heme enzymes in plants. Environ. Sci. Pollut. Res. 2016, 23, 19002-19029. [CrossRef] [PubMed]

6. Kaushal, J.; Mehandia, S.; Singh, G.; Raina, A.; Arya, S.K. Catalase enzyme: Application in bioremediation and food industry. Biocatal. Agric. Biotechnol. 2018, 16, 192-199. [CrossRef]

7. GONZÁLEZ, E. The C-terminal domain of plant catalases Implications for a glyoxysomal targeting sequence. Eur. J. Biochem. 1991, 199, 211-215. [CrossRef] [PubMed]

8. Drory, A.; Woodson, W.R. Molecular cloning and nucleotide sequence of a cDNA encoding catalase from tomato. Plant Physiol. 1992, 100, 1605. [CrossRef]

9. Chen, H.-J.; Wu, S.-D.; Huang, G.-J.; Shen, C.-Y.; Afiyanti, M.; Li, W.-J.; Lin, Y.-H. Expression of a cloned sweet potato catalase SPCAT1 alleviates ethephon-mediated leaf senescence and $\mathrm{H}_{2} \mathrm{O}_{2}$ elevation. J. Plant Physiol. 2012, 169, 86-97. [CrossRef] [PubMed]

10. Skadsen, R.W.; Schulze-Lefert, P.; Herbst, J.M. Molecular cloning, characterization and expression analysis of two catalase isozyme genes in barley. Plant Mol. Biol. 1995, 29, 1005-1014. [CrossRef]

11. Willekens, H.; Villarroel, R.; Van Montagu, M.; Inzé, D.; Van Camp, W. Molecular identification of catalases from Nicotiana plumbaginifolia (L.). FEBS Lett. 1994, 352, 79-83. [CrossRef]

12. Guan, L.; Scandalios, J.G. Developmentally related responses of maize catalase genes to salicylic acid. Proc. Nat. Acad. Sci. USA 1995, 92, 5930-5934. [CrossRef] [PubMed]

13. Esaka, M.; Yamada, N.; Kitabayashi, M.; Setoguchi, Y.; Tsugeki, R.; Kondo, M.; Nishimura, M. cDNA cloning and differential gene expression of three catalases in pumpkin. Plant Mol. Biol. 1997, 33, 141-155. [CrossRef] [PubMed]

14. Du, Y.Y.; Wang, P.C.; Chen, J.; Song, C.P. Comprehensive functional analysis of the catalase gene family in Arabidopsis thaliana. J. Integr. Plant Biol. 2008, 50, 1318-1326. [CrossRef] [PubMed] 
15. Joo, J.; Lee, Y.H.; Song, S.I. Rice CatA, CatB, and CatC are involved in environmental stress response, root growth, and photorespiration, respectively. J. Plant Biol. 2014, 57, 375-382. [CrossRef]

16. Hu, L.; Yang, Y.; Jiang, L.; Liu, S. The catalase gene family in cucumber: Genome-wide identification and organization. Genet. Mol. Biol. 2016, 39, 408-415. [CrossRef] [PubMed]

17. Alam, N.B.; Ghosh, A. Comprehensive analysis and transcript profiling of Arabidopsis thaliana and Oryza sativa catalase gene family suggests their specific roles in development and stress responses. Plant Physiol. Biochem. 2018, 123, 54-64. [CrossRef]

18. Wang, W.; Cheng, Y.; Chen, D.; Liu, D.; Hu, M.; Dong, J.; Zhang, X.; Song, L.; Shen, F. The catalase gene family in cotton: Genome-wide characterization and bioinformatics analysis. Cells 2019, 8, 86. [CrossRef]

19. Inaba, J.-I.; Kim, B.M.; Shimura, H.; Masuta, C. Virus-induced necrosis is a consequence of direct protein-protein interaction between a viral RNA-silencing suppressor and a host catalase. Plant Physiol. 2011, 156, 2026-2036. [CrossRef]

20. Yong, B.; Wang, X.; Xu, P.; Zheng, H.; Fei, X.; Hong, Z.; Ma, Q.; Miao, Y.; Yuan, X.; Jiang, Y.; et al. Isolation and abiotic stress resistance analyses of a catalase gene from Ipomoea batatas (L.) Lam. BioMed Res. Inter. 2017, 2017. [CrossRef] [PubMed]

21. Su, Y.; Guo, J.; Ling, H.; Chen, S.; Wang, S.; Xu, L.; Allan, A.C.; Que, Y. Isolation of a novel peroxisomal catalase gene from sugarcane, which is responsive to biotic and abiotic stresses. PLoS ONE 2014, 9, e84426. [CrossRef]

22. Balestrasse, K.B.; Yannarelli, G.G.; Noriega, G.O.; Batlle, A.; Tomaro, M.L. Heme oxygenase and catalase gene expression in nodules and roots of soybean plants subjected to cadmium stress. Biometals 2008, 21, 433-441. [CrossRef]

23. Lin, K.-H.; Huang, H.-C.; Lin, C.-Y. Cloning, expression and physiological analysis of broccoli catalase gene and Chinese cabbage ascorbate peroxidase gene under heat stress. Plant Cell Rep. 2010, 29, 575-593. [CrossRef] [PubMed]

24. Queval, G.; Issakidis-Bourguet, E.; Hoeberichts, F.A.; Vandorpe, M.; Gakière, B.; Vanacker, H.; Miginiac-Maslow, M.; Van Breusegem, F.; Noctor, G. Conditional oxidative stress responses in the Arabidopsis photorespiratory mutant cat2 demonstrate that redox state is a key modulator of daylength-dependent gene expression, and define photoperiod as a crucial factor in the regulation of $\mathrm{H}_{2} \mathrm{O}_{2}$-induced cell death. Plant J. 2007, 52, 640-657.

25. Contento, A.L.; Bassham, D.C. Increase in catalase-3 activity as a response to use of alternative catabolic substrates during sucrose starvation. Plant Physiol. Biochem. 2010, 48, 232-238. [CrossRef] [PubMed]

26. Polidoros, A.; Mylona, P.; Scandalios, J. Transgenic tobacco plants expressing the maize Cat2 gene have altered catalase levels that affect plant-pathogen interactions and resistance to oxidative stress. Trans. Res. 2001, 10, 555-569. [CrossRef] [PubMed]

27. Lee, S.H.; An, C.S. Differential expression of three catalase genes in hot pepper (Capsicum annuum L.). Mol. Cells 2005, 20, 247-255.

28. Niewiadomska, E.; Polzien, L.; Desel, C.; Rozpadek, P.; Miszalski, Z.; Krupinska, K. Spatial patterns of senescence and development-dependent distribution of reactive oxygen species in tobacco (Nicotiana tabacum) leaves. J. Plant Physiol. 2009, 166, 1057-1068. [CrossRef] [PubMed]

29. Vuosku, J.; Sutela, S.; Kestilä, J.; Jokela, A.; Sarjala, T.; Häggman, H. Expression of catalase and retinoblastoma-related protein genes associates with cell death processes in Scots pine zygotic embryogenesis. BMC Plant Biol. 2015, 15, 88. [CrossRef]

30. Cui, C.; Wang, J.-J.; Zhao, J.-H.; Fang, Y.-Y.; He, X.-F.; Guo, H.-S.; Duan, C.-G. A Brassica miRNA Regulates Plant Growth and Immunity through Distinct Modes of Action. Mol. Plant 2020, 13, 231-245. [CrossRef]

31. Khandal, H.; Parween, S.; Roy, R.; Meena, M.K.; Chattopadhyay, D. MicroRNA profiling provides insights into post-transcriptional regulation of gene expression in chickpea root apex under salinity and water deficiency. Sci. Rep. 2017, 7, 1-14. [CrossRef]

32. Ding, Y.; Ding, L.; Xia, Y.; Wang, F.; Zhu, C. Emerging roles of microRNAs in plant heavy metal tolerance and homeostasis. J. Agric. Food Chem. 2020, 68, 1958-1965. [CrossRef] [PubMed]

33. Ravichandran, S.; Ragupathy, R.; Edwards, T.; Domaratzki, M.; Cloutier, S. MicroRNA-guided regulation of heat stress response in wheat. BMC Genom. 2019, 20, 488. [CrossRef]

34. Park, S.-Y.; Grabau, E. Bypassing miRNA-mediated gene regulation under drought stress: Alternative splicing affects CSD1 gene expression. Plant Mol. Biol. 2017, 95, 243-252. [CrossRef]

35. SHI, G.-q.; FU, J.-y.; RONG, L.-j.; ZHANG, P.-y.; GUO, C.-j.; Kai, X. TaMIR1119, a miRNA family member of wheat (Triticum aestivum), is essential in the regulation of plant drought tolerance. J. Integr. Agric. 2018, 17, 2369-2378. [CrossRef]

36. Ci, D.; Song, Y.; Tian, M.; Zhang, D. Methylation of miRNA genes in the response to temperature stress in Populus simonii. Front. Plant Sci. 2015, 6, 921. [CrossRef]

37. Raza, A. Eco-physiological and Biochemical Responses of Rapeseed (Brassica napus L.) to Abiotic Stresses: Consequences and Mitigation Strategies. J. Plant Growth Regul. 2020. [CrossRef]

38. Raza, A.; Hafeez, M.B.; Zahra, N.; Shaukat, K.; Umbreen, S.; Tabassum, J.; Charagh, S.; Khan, R.S.A.; Hasanuzzaman, M. The Plant Family Brassicaceae: Introduction, Biology, and Importance. In The Plant Family Brassicaceae; Hasanuzzaman, M., Ed.; Springer: Singapore, 2020; pp. 1-43.

39. He, H.; Lei, Y.; Yi, Z.; Raza, A.; Zeng, L.; Yan, L.; Xiaoyu, D.; Yong, C.; Xiling, Z. Study on the mechanism of exogenous serotonin improving cold tolerance of rapeseed (Brassica napus L.) seedlings. Plant Growth Regul. 2021. [CrossRef]

40. Song, J.-M.; Guan, Z.; Hu, J.; Guo, C.; Yang, Z.; Wang, S.; Liu, D.; Wang, B.; Lu, S.; Zhou, R.; et al. Eight high-quality genomes reveal pan-genome architecture and ecotype differentiation of Brassica napus. Nat. Plants 2020, 6, 34-45. [CrossRef] [PubMed]

41. Cavell, A.C.; Lydiate, D.; Parkin, I.; Dean, C.; Trick, M. Collinearity between a 30-centimorgan segment of Arabidopsis thaliana chromosome 4 and duplicated regions within the Brassica napus genome. Genome 1998, 41, 62-69. [CrossRef] [PubMed]

42. Frugoli, J.A.; McPeek, M.A.; Thomas, T.L.; McClung, C.R. Intron loss and gain during evolution of the catalase gene family in angiosperms. Genetics 1998, 149, 355-365. [CrossRef] [PubMed] 
43. Zou, J.-J.; Li, X.-D.; Ratnasekera, D.; Wang, C.; Liu, W.-X.; Song, L.-F.; Zhang, W.-Z.; Wu, W.-H. Arabidopsis CALCIUMDEPENDENT PROTEIN KINASE8 and CATALASE3 function in abscisic acid-mediated signaling and $\mathrm{H}_{2} \mathrm{O}_{2}$ homeostasis in stomatal guard cells under drought stress. Plant Cell 2015, 27, 1445-1460. [CrossRef]

44. Hackenberg, T.; Juul, T.; Auzina, A.; Gwiżdż, S.; Małolepszy, A.; Van Der Kelen, K.; Dam, S.; Bressendorff, S.; Lorentzen, A.; Roepstorff, P. Catalase and NO CATALASE ACTIVITY1 promote autophagy-dependent cell death in Arabidopsis. Plant Cell 2013, 25, 4616-4626. [CrossRef]

45. Corpas, F.J.; Barroso, J.B.; González-Gordo, S.; Muñoz-Vargas, M.A.; Palma, J.M. Hydrogen sulfide: A novel component in Arabidopsis peroxisomes which triggers catalase inhibition. J. Integr. Plant Biol. 2019, 61, 871-883. [CrossRef]

46. Li, J.; Liu, J.; Wang, G.; Cha, J.-Y.; Li, G.; Chen, S.; Li, Z.; Guo, J.; Zhang, C.; Yang, Y. A chaperone function of NO CATALASE ACTIVITY1 is required to maintain catalase activity and for multiple stress responses in Arabidopsis. Plant Cell 2015, 27, 908-925. [CrossRef]

47. Chiang, C.-M.; Chen, S.-P.; Chen, L.-F.O.; Chiang, M.-C.; Chien, H.-L.; Lin, K.-H. Expression of the broccoli catalase gene (BoCAT) enhances heat tolerance in transgenic Arabidopsis. J. Plant Biochem. Biotechnol. 2014, 23, 266-277. [CrossRef]

48. Zhou, Y.-B.; Liu, C.; Tang, D.-Y.; Yan, L.; Wang, D.; Yang, Y.-Z.; Gui, J.-S.; Zhao, X.-Y.; Li, L.-G.; Tang, X.-D.; et al. The receptor-like cytoplasmic kinase STRK1 phosphorylates and activates CatC, thereby regulating $\mathrm{H}_{2} \mathrm{O}_{2}$ homeostasis and improving salt tolerance in rice. Plant Cell 2018, 30, 1100-1118. [CrossRef] [PubMed]

49. Liu, J.; Cui, L.; Xie, Z.; Zhang, Z.; Liu, E.; Peng, X. Two NCA1 isoforms interact with catalase in a mutually exclusive manner to redundantly regulate its activity in rice. BMC Plant Biol. 2019, 19, 105. [CrossRef]

50. Shahpiri, A.; Deljoonia, R. Enhancement of catalase and superoxide dismutase activities in transgenic Escherichia coli expressing rice metallothionein isoforms. J. BioSci. Biotechnol. 2018, 7, 5-10.

51. Matsumura, T.; Tabayashi, N.; Kamagata, Y.; Souma, C.; Saruyama, H. Wheat catalase expressed in transgenic rice can improve tolerance against low temperature stress. Physiol. Plant 2002, 116, 317-327. [CrossRef]

52. Hameed, A.; Naseer, S.; Iqbal, T.; Syed, H.; Haq, M.A. Effects of $\mathrm{NaCl}$ salinity on seedling growth, senescence, catalase and protease activities in two wheat genotypes differing in salt tolerance. Pak. J. Bot. 2008, 40, 1043-1051.

53. Srivalli, B.; Khanna-Chopra, R. Induction of new isoforms of superoxide dismutase and catalase enzymes in the flag leaf of wheat during monocarpic senescence. Biochem. Biophys. Res. Commun. 2001, 288, 1037-1042. [CrossRef]

54. dos Santos, C.M.; de Almeida Silva, M.; Lima, G.P.P.; Bortolheiro, F.P.d.A.P.; Brunelli, M.C.; de Holanda, L.A.; Oliver, R. Physiological changes associated with antioxidant enzymes in response to sugarcane tolerance to water deficit and rehydration. Sugar Tech. 2015, 17, 291-304. [CrossRef]

55. Liu, Y.; Hu, X.; Yao, Y.; Xu, L.; Xing, S. Isolation and expression analysis of catalase genes in Erianthus arundinaceus and sugarcane. Sugar Tech. 2016, 18, 468-477. [CrossRef]

56. Zhou, Y.; Liu, S.; Yang, Z.; Yang, Y.; Jiang, L.; Hu, L. CsCAT3, a catalase gene from Cucumis sativus, confers resistance to a variety of stresses to Escherichia coli. Biotechnol. Biotechnol. Equip. 2017, 31, 886-896. [CrossRef]

57. Murota, K.; Shimura, H.; Takeshita, M.; Masuta, C. Interaction between Cucumber mosaic virus $2 \mathrm{~b}$ protein and plant catalase induces a specific necrosis in association with proteasome activity. Plant Cell Rep. 2017, 36, 37-47. [CrossRef] [PubMed]

58. Wang, Q.; Li, C.X.; Fan, X.; Wang, P.; Cui, L. Immobilization of catalase on cotton fabric oxidized by sodium periodate. Biocatal. Biotrans. 2008, 26, 437-443. [CrossRef]

59. Luo, X.; Wu, J.; Li, Y.; Nan, Z.; Guo, X.; Wang, Y.; Zhang, A.; Wang, Z.; Xia, G.; Tian, Y. Synergistic effects of GhSOD1 and GhCAT1 overexpression in cotton chloroplasts on enhancing tolerance to methyl viologen and salt stresses. PLoS ONE 2013, 8, e54002. [CrossRef] [PubMed]

60. Duman, Y.A.; Kaya, E. Three-phase partitioning as a rapid and easy method for the purification and recovery of catalase from sweet potato tubers (Solanum tuberosum). Appl. Biochem. Biotechnol. 2013, 170, 1119-1126. [CrossRef]

61. Afiyanti, M.; Chen, H.-J. Catalase activity is modulated by calcium and calmodulin in detached mature leaves of sweet potato. J. Plant Physiol. 2014, 171, 35-47. [CrossRef]

62. Xing, Y.; Jia, W.; Zhang, J. AtMEK1 mediates stress-induced gene expression of $C A T 1$ catalase by triggering $\mathrm{H}_{2} \mathrm{O}_{2}$ production in Arabidopsis. J. Exp. Bot. 2007, 58, 2969-2981. [CrossRef]

63. Guo, P.; Li, Z.; Huang, P.; Li, B.; Fang, S.; Chu, J.; Guo, H. A tripartite amplification loop involving the transcription factor WRKY75, salicylic acid, and reactive oxygen species accelerates leaf senescence. Plant Cell 2017, 29, 2854-2870. [CrossRef]

64. Corpas, F.J.; Barroso, J.B. Lead-induced stress, which triggers the production of nitric oxide (NO) and superoxide anion (O2*-) in Arabidopsis peroxisomes, affects catalase activity. Nitric Oxide 2017, 68, 103-110. [CrossRef]

65. Vighi, I.; Benitez, L.; do Amaral, M.; Auler, P.; Moraes, G.; Rodrigues, G.; Da Maia, L.; Pinto, L.; Braga, E. Changes in gene expression and catalase activity in Oryza sativa L. under abiotic stress. Genet. Mol. Res. 2016, 15, gmr15048977. [CrossRef] [PubMed]

66. Wang, W.; Liu, D.; Zhang, X.; Chen, D.; Cheng, Y.; Shen, F. Plant microRNAs in cross-kingdom regulation of gene expression. Int. J. Mol. Sci. 2018, 19, 2007. [CrossRef] [PubMed]

67. Huang, S.Q.; Xiang, A.L.; Che, L.L.; Chen, S.; Li, H.; Song, J.B.; Yang, Z.M. A set of miRNAs from Brassica napus in response to sulphate deficiency and cadmium stress. Plant Biotechnol. J. 2010, 8, 887-899. [CrossRef]

68. Zhou, Z.S.; Song, J.B.; Yang, Z.M. Genome-wide identification of Brassica napus microRNAs and their targets in response to cadmium. J. Exp. Bot. 2012, 63, 4597-4613. [CrossRef] [PubMed] 
69. Chen, L.; Chen, L.; Zhang, X.; Liu, T.; Niu, S.; Wen, J.; Yi, B.; Ma, C.; Tu, J.; Fu, T. Identification of miRNAs that regulate silique development in Brassica napus. Plant Sci. 2018, 269, 106-117. [CrossRef]

70. Buhtz, A.; Springer, F.; Chappell, L.; Baulcombe, D.C.; Kehr, J. Identification and characterization of small RNAs from the phloem of Brassica napus. Plant J. 2008, 53, 739-749. [CrossRef] [PubMed]

71. Fu, Y.; Mason, A.S.; Zhang, Y.; Lin, B.; Xiao, M.; Fu, D.; Yu, H. MicroRNA-mRNA expression profiles and their potential role in cadmium stress response in Brassica napus. BMC Plant Biol. 2019, 19, 1-20. [CrossRef] [PubMed]

72. Körbes, A.P.; Machado, R.D.; Guzman, F.; Almerao, M.P.; de Oliveira, L.F.V.; Loss-Morais, G.; Turchetto-Zolet, A.C.; Cagliari, A.; dos Santos Maraschin, F.; Margis-Pinheiro, M. Identifying conserved and novel microRNAs in developing seeds of Brassica napus using deep sequencing. PLoS ONE 2012, 7, e50663. [CrossRef] [PubMed]

73. Shen, E.; Zou, J.; Hubertus Behrens, F.; Chen, L.; Ye, C.; Dai, S.; Li, R.; Ni, M.; Jiang, X.; Qiu, J. Identification, evolution, and expression partitioning of miRNAs in allopolyploid Brassica napus. J. Exp. Bot. 2015, 66, 7241-7253. [CrossRef] [PubMed]

74. Casati, P. Analysis of UV-B regulated miRNAs and their targets in maize leaves. Plant Signal. Behav. 2013, 8, e26758. [CrossRef] [PubMed]

75. Li, S.; Yu, X.; Lei, N.; Cheng, Z.; Zhao, P.; He, Y.; Wang, W.; Peng, M. Genome-wide identification and functional prediction of cold and/or drought-responsive lncRNAs in cassava. Sci. Rep. 2017, 7, 45981. [CrossRef] [PubMed]

76. Nageshbabu, R.; Jyothi, M.; Sharadamma, N. Expression of miRNAs regulates growth and development of French bean (Phaseolus vulgaris) under salt and drought stress conditions. ISCA J. Biol. Sci. 2013, 2, 52-56.

77. Zhang, Y.; Zhu, X.; Chen, X.; Song, C.; Zou, Z.; Wang, Y.; Wang, M.; Fang, W.; Li, X. Identification and characterization of cold-responsive microRNAs in tea plant (Camellia sinensis) and their targets using high-throughput sequencing and degradome analysis. BMC Plant Biol. 2014, 14, 271. [CrossRef]

78. Yang, Z.; Wang, Z.; Yang, C.; Yang, Z.; Li, H.; Wu, Y. Physiological responses and small RNAs changes in maize under nitrogen deficiency and resupply. Genes Genom. 2019, 41, 1183-1194. [CrossRef]

79. Wang, Z.; Chen, Y.; Fang, H.; Shi, H.; Chen, K.; Zhang, Z.; Tan, X. Selection of reference genes for quantitative reversetranscription polymerase chain reaction normalization in Brassica napus under various stress conditions. Mol. Genet. Genom. 2014, 289, 1023-1035. [CrossRef] [PubMed] 